\title{
Weak and strong dynamic scaling in a one-dimensional driven coupled-field model: Effects of kinematic waves
}

\author{
Dibyendu Das, ${ }^{1, *}$ Abhik Basu, ${ }^{2,3, \dagger}$ Mustansir Barma, ${ }^{1,4, \$}$ and Sriram Ramaswamy ${ }^{3,4, \S}$ \\ ${ }^{1}$ Department of Theoretical Physics, Tata Institute of Fundamental Research, Homi Bhabha Road, Mumbai 400005, India \\ ${ }^{2}$ Poornaprajna Institute of Scientific Research, Bangalore, India \\ ${ }^{3}$ Centre for Condensed Matter Theory, Department of Physics, Indian Institute of Science, Bangalore 560012, India \\ ${ }^{4}$ Jawaharlal Nehru Center for Advanced Scientific Research, Bangalore, India
}

\begin{abstract}
We study the coupled dynamics of the displacement fields in a one-dimensional coupled-field model for drifting crystals, first proposed by Lahiri and Ramaswamy [Phys. Rev. Lett. 79, 1150 (1997)]. We present some exact results for the steady state and the current in the lattice version of the model for a special subspace in the parameter space, within the region where the model displays kinematic waves. We use these results to construct the effective continuum equations corresponding to the lattice model. These equations decouple at the linear level in terms of the eigenmodes. We examine the long-time, large-distance properties of the correlation functions of the eigenmodes by using symmetry arguments, Monte Carlo simulations, and self-consistent mode-coupling methods. For most parameter values, the scaling exponents of the Kardar-Parisi-Zhang equation are obtained. However, for certain symmetry-determined values of the coupling constants the two eigenmodes, although nonlinearly coupled, are characterized by two distinct dynamic exponents. We discuss the possible application of the dynamic renormalization group in this context.
\end{abstract}

\section{INTRODUCTION}

\section{A. Background}

Spatial and temporal correlations in spatially extended systems with a conservation law or a continuous invariance are widely observed to satisfy a scaling or homogeneity property. For instance, if the system is described by a single scalar field $\phi(x, t)$, the correlation function $C(x, t)$ $\equiv\langle\phi(0,0) \phi(x, t)\rangle$ satisfies

$$
C(x, t) \approx b^{2 \chi} C\left(b x, b^{z} t\right)
$$

under rescaling of space by a factor $b$. Here $z$ is the dynamic exponent while $\chi$ describes the spatial scaling of the field. Equation (1) holds in the rest frame of the $\phi$ fluctuations, so that if the system has wavelike excitations, e.g., kinematic waves [1] of moving $\phi$ fluctuations, it is necessary to perform a Galilean shift to comove with the wave. The exponent $z$ then describes the dissipation of the fluctuation wave, with a fluctuation of spatial extent $\Delta x$ having a lifetime proportional to $(\Delta x)^{z}$.

Now consider the scaling properties of systems with several coupled fields, say $\left\{\phi_{\alpha}, \alpha=1-N\right\}$, whose dynamical evolution involves interfield couplings both at the linear and nonlinear levels that result in propagating kinematic waves. At the linear level, the problem requires diagonalizing the matrix of couplings. The eigenvectors $\psi_{\mu}$ involve linear

\footnotetext{
*Present address: Martin Fisher School of Physics, Brandeis University, Mailstop 057, Waltham, MA 02454-9110. Email address: dibyendu@octane.cc.brandeis.edu

${ }^{\dagger}$ Email address: abhik@physics.iisc.ernet.in

*Email address: barma@theory.tifr.res.in

${ }^{\S}$ Email address: sriram@physics.iisc.ernet.in
}

combinations of the $\phi_{\alpha}$ and represent modes that propagate as independent kinematic waves. The real and imaginary parts of the eigenvalues $c_{\mu}$ encode, respectively, the speeds and dampings of the corresponding kinematic waves and, in general, differ from one wave to another. By performing a Galilean shift with speed $c_{\mu}$, one may move to the rest frame of mode $\mu$; kinematic waves corresponding to other modes are not stationary in this frame, however, and these moving modes also contribute to the dissipation of mode $\mu$ as they are coupled nonlinearly to it. The correlation function $C_{\mu}(x, t) \equiv\left\langle\psi_{\mu}(0,0) \psi_{\mu}(x, t)\right\rangle$ is expected to satisfy

$$
C_{\mu}(x, t) \approx b^{2 \chi_{\mu}} C\left(b x, b^{z} \mu t\right) .
$$

where $\chi_{\mu}$ characterizes the spatial scaling of mode $\mu$ and $z_{\mu}$ is the corresponding dynamic exponent.

A question arises: Is there a single common value $z$ that characterizes the decay of all the modes $\mu$ ? When the answer is yes, as in fact it generally is, we say that the system obeys strong dynamic scaling. Considerable interest is therefore attached to exceptions of this general rule. Accordingly, one would like to characterize the conditions for the occurrence of weak dynamic scaling, when at least one $z_{\mu}$ is different from the rest. A priori, there are two sets of circumstances when weak dynamic scaling may be expected.

(i) If the transformation from $\phi_{\alpha}$ to $\psi_{\mu}$, which is designed to decouple modes at the linear level, actually succeeds in decoupling them for the full nonlinear problem, then evidently each mode evolves autonomously and independent $z_{\mu}$ 's may arise. In fact, a complete decoupling at the nonlinear level does occur in the context of a reduced model of magnetohydrodynamic (MHD) turbulence [2], and may well arise in other problems as well. In the MHD case, however, it turns out that both modes obey evolution equations with 
similar (autonomous) nonlinearites, so that a common value of $z$ ensues. But this need not be the case for other problems.

(ii) Consider a situation in which the evolution of a subset of the fields, say $\left\{u_{\beta}\right\}$, does not involve the others $\left\{u_{\alpha}\right\}$, while the evolution of the set $\left\{u_{\alpha}\right\}$ does involve $\left\{u_{\beta}\right\}$. In this case, $z_{\beta}$ and $z_{\alpha}$ may take on distinct values. Indeed, this is borne out by numerical studies of two-field dynamics [3], which show that weak scaling occurs if the evolution is autonomuous in one of the two fields, or very nearly so, in which case strong crossover effects may be expected.

One of the principal results of this paper, which we present below, is a third possible scenario for weak dynamical scaling, where neither field is autonomous.

\section{B. Results}

In this paper we examine weak and strong dynamical scaling in a system with two coupled fields, which result in two coupled kinematic waves characterized by mode coordinates $\psi_{1}$ and $\psi_{2}$, respectively. We work both with a lattice model involving two sets of spins and with the corresponding continuum equations involving two coupled scalar fields. The analysis of the lattice model is facilitated by showing that along certain representative loci in parameter space, the steady state has a product measure form. This allows the current to be found and a continuum expansion to be made with coefficients that explicitly involve the parameters and mean occupations of the lattice model. This enables us to make direct comparisons between the results of numerical simulations of the lattice model and analytical self-consistent calculations for the continuum equations.

Our most interesting result is the identification of a third set of circumstances beyond (i) and (ii) mentioned in Sec. IA, in which weak dynamic scaling results despite each mode being nonlinearly coupled to the other. This involves symmetry properties of the kinematic waves: we find weak dynamic scaling if we choose model parameters so that the evolution equations are invariant under inversion of the second mode coordinate $\left(\psi_{2} \rightarrow-\psi_{2}\right)$ but not under $\psi_{1} \rightarrow-\psi_{1}$. In that case our numerical simulations show that $z_{1}=3 / 2$, while $z_{2}=2$ with multiplicative logarithmic corrections. We also study the problem within a self-consistent modecoupling calculation, which shows that the different dynamic exponents arise in a rather interesting way: the linearized version of the problem has $z=2$ for both fields. The scattering of $\psi_{1}$ by fluctuations in $\psi_{2}$ and vice versa gives rise to singular corrections to the diffusivity for $\psi_{1}$, leading to $z_{1}$ $=3 / 2$. The fluctuations, however, cause no singular correction to the relaxation of $\psi_{2}$, leaving $z_{2}=2$. For most other parameter values, however, the evolution equations are not invariant under changing the sign of either $\psi_{1}$ or $\psi_{2}$, and we find the more common strong dynamic scaling with $z_{1}=z_{2}$ $=3 / 2$.

The remainder of this paper is organized as follows. In Sec. II, we review briefly the continuum stochastic dynamical equations of Ref. [4]. In Sec. III we present the lattice model and show how the condition of pairwise balance can be used to find the exact steady state if the transition rates satisfy a certain relation. In Sec. IV we characterize changes of the symmetry of the evolution equations as overall densities are varied and report the results of extensive numerical simulations, which show that the values of the dynamic exponents depend strongly on these symmetries. In particular, we present evidence for weak dynamic scaling when the two kinematic waves have different symmetries. In Sec. V we describe analytical methods, primarily a one-loop selfconsistent treatment of the continuum stochastic partial differential equation (PDEs), for calculating the exponents in the weak dynamic scaling regime. We also outline a dynamic renormalization-group procedure for this regime, discuss the difficulties that arise therein, and remind the reader how strong dynamic scaling is restored for generic values of the parameters in the model. We close in Sec. VI with a summary.

\section{CONTINUUM STOCHASTIC PDEs FOR DRIFTING CRYSTALS}

We review very briefly here the construction of our model equations of motion; details may be found in $[4,5]$. The physical system that inspired the initial work on the model was a lattice drifting through a dissipative medium. There are at least two examples of this: (i) steadily sedimenting colloidal crystals and (ii) a flux lattice driven by the action of the Lorentz force of an imposed supercurrent through a type II superconductor. If inertia is ignored, the equation of motion of the displacement field $\mathbf{u}(\mathbf{r}, t)$ is of the form velocity $=$ mobility $\times$ force, i.e.,

$$
\dot{\mathbf{u}}=\mathbf{M}(\boldsymbol{\nabla} \mathbf{u})(\mathbf{D} \nabla \nabla \mathbf{u}+\mathbf{F})+\zeta,
$$

where the mobility tensor $\mathbf{M}$ is allowed to depend on the lattice distortion $\nabla \mathbf{u}$, the tensor $\mathbf{D}$ represents elastic forces, $\mathbf{F}$ is the driving force, and $\zeta$ is a suitable noise source. Our results are for a highly simplified model with the same physics as in Eq. (3). This model, constructed and studied in [4,5], describes the coupled dynamics of two fields $u_{x}$ and $u_{z}$ (the displacements transverse to and along the drift direction, respectively) as a function of one coordinate $x$ transverse to the drift direction $\hat{\mathbf{z}}$. The equations of motion are

$$
\begin{gathered}
\dot{u}_{x}=\lambda_{12} \partial_{x} u_{z}+\gamma_{1} \partial_{x} u_{x} \partial_{x} u_{z}+D_{1} \partial_{x}^{2} u_{x}+f_{x}, \\
\dot{u}_{z}=\lambda_{21} \partial_{x} u_{x}+\gamma_{2}\left(\partial_{x} u_{x}\right)^{2}+\gamma_{3}\left(\partial_{x} u_{z}\right)^{2}+D_{2} \partial_{x}^{2} u_{z}+f_{z},
\end{gathered}
$$

where $f_{x}$ and $f_{z}$ are zero-mean, Gaussian, spatiotemporally white noise sources. The equations are invariant under the joint operations $x \rightarrow-x, u_{x} \rightarrow-u_{x}$. For $\lambda_{12}=\lambda_{21}=0$, Eqs. (4) and (5) reduce to the Ertaş-Kardar (EK) [3] equations for drifting polymers with the larger symmetry $x \rightarrow-x$ (with or without $u_{x} \rightarrow-u_{x}$ ). The system can distinguish between up and down: there is no invariance under inversion of $u_{z}$. The terms in Eqs. (4) and (5) involving first spatial derivatives have the following interpretation: a tilt $\left(\partial_{x} u_{z}\right)$ produces a lateral drift (at a rate that depends on the density perturbations $\partial_{x} u_{x}$ ), while the vertical speed depends both on com- 
pressions or dilations $\left(\partial_{x} u_{x}\right)$ as well as tilts $\left(\partial_{x} u_{z}\right)$. In this paper we shall consider only the case $\lambda_{12} \lambda_{21}>0$, in which case the dispersion relation

$$
\omega= \pm \sqrt{\lambda_{12} \lambda_{21}} q-i D q^{2}
$$

holds for the linearized version of Eqs. (4) and (5) and predicts traveling waves at a small wave number $q$. The cases $\lambda_{12} \lambda_{21} \leqslant 0$ have been discussed extensively elsewhere [5-7]. The mode coordinates corresponding to Eq. (6) are given by

$$
\psi_{1,2} \equiv \sqrt{c} u_{x} \pm \sqrt{b} u_{z} .
$$

As discussed in [5], the long-time, large-length-scale behavior of the PDEs (4) and (5) are expected to be the same as those of a particular two-species Ising-Kawasaki model in which the jump rate of each species depends on the local density of the other. We turn next to this discrete model and its dynamics.

\section{THE LATTICE MODEL}

The lattice model is defined in terms of two sets of variables $\left\{\sigma_{i}\right\}$ and $\left\{\tau_{i-1 / 2}\right\}$ that reside on two interpenetrating sublattices with periodic boundary conditions; the former set occupies the integer sites and the latter occupies the halfinteger midbond locations of a one-dimensional lattice with $L$ sites. Each $\sigma_{i}$ and $\tau_{i-1 / 2}$ is an Ising variable taking on values \pm 1 . They represent discrete versions of the density and tilt fields in the sedimentation problem: If $\sigma_{i}$ is 1 , there is a particle (+) at site $i$, and if $\sigma_{i}=-1$, there is no particle $(-)$. The variable $\tau_{i-1 / 2}=1$ and -1 , implies two values / and $\backslash$ of the local tilt, respectively. A typical configuration of the full system is thus $+\backslash-/-I+\backslash-/+/+/+\backslash-$.

Both sets of variables are conserved, i.e., $\Sigma \sigma_{i}$ and $\Sigma \tau_{i-1 / 2}$ and the associated densities $\rho_{1}^{o}=\Sigma\left(1+\sigma_{i}\right) / 2 L$ and $\rho_{2}^{o}$ $=\Sigma\left(1+\tau_{i-1 / 2}\right) / 2 L$ are constant. We consider a $\tau$-dependent local field that guides the $\sigma$ current and vice versa. Thus, for instance, the Kawasaki exchange dynamics of the adjacent spins $\sigma_{i}$ and $\sigma_{i+1}$ occurs at a rate that depends on $\tau_{i+1 / 2}$. The moves and the corresponding rates are depicted below,

$$
\begin{aligned}
& \text { (1) }+\backslash-\rightarrow-\backslash+r_{1}, \\
& \text { (2) }-\backslash+\rightarrow+\backslash-r_{2}, \\
& \text { (3) }-/+\rightarrow+/-r_{1}, \\
& \text { (4) }+/-\rightarrow-/+r_{2}, \\
& \text { (5) } /-\backslash \rightarrow \backslash-/ p_{2}, \\
& \text { (6) } \backslash-/ \rightarrow /-\backslash p_{1}, \\
& \text { (7) } \backslash+/ \rightarrow /+\backslash p_{2}, \\
& \text { (8) } /+\backslash \rightarrow \backslash+/ p_{1} .
\end{aligned}
$$

The macroscopic behavior of the model is determined by the relative values of the rates; a brief review of the phases

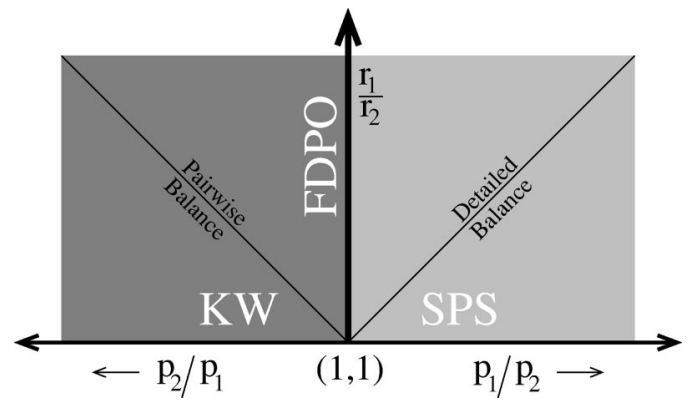

FIG. 1. The phase diagram of the Lahiri-Ramaswamy model. The strongly phase separated phase (SPS) is separated from the kinematic wave phase $(\mathrm{KW})$ of interest in this paper by the threshold line $p_{1}=p_{2}$ along which there is fluctuation-dominated phase ordering. The steady state can be found exactly along the line $p_{2} / p_{1}=r_{1} / r_{2}$, by using the condition of pairwise balance.

and their characteristics appears in [6]. There are two distinct regimes separated by a nonequilibrium phase boundary as depicted in Fig. 1. The regime $p_{1}>p_{2}$, marked SPS in Fig. 1 was explored in detail in [5]. In this phase, the system undergoes spontaneous phase separation of a particularly strong sort. Along the boundary $p_{1}=p_{2}$, marked FDPO in Fig. 1, the system undergoes fluctuation-dominated phase ordering of a delicate sort as discussed in [7]. Finally, in the phase of the model with $p_{1}<p_{2}$, marked $\mathrm{KW}$ in Fig. 1, there is no phase separation and fluctuations are transported by kinematic waves. This is the regime of interest in this paper.

\section{A. Exact results for the steady state}

The steady state can be found exactly provided that $r_{1}$ $=p_{2}$ and $r_{2}=p_{1}$ (along the line marked pairwise balance in Fig. 1). To this end, let us choose new symbols to denote the values of the variables $\sigma$ and $\tau$ : use 1 if a site or bond is occupied by a + or $/$, and use 0 for - or $\backslash$. Then the moves $(1)-(8)$ listed above reduce to moves $(a),(b),(\bar{a}),(\bar{b})$ as follows:

$$
\begin{aligned}
& (1),(5) \Rightarrow(a): 100 \rightarrow 001 p_{2}, \\
& (3),(7) \Rightarrow(b): 011 \rightarrow 110 p_{2}, \\
& (2),(6) \Rightarrow(\bar{a}): 001 \rightarrow 100 p_{1}, \\
& (4),(8) \Rightarrow(\bar{b}): 110 \rightarrow 011 p_{1} .
\end{aligned}
$$

The use of the new symbols 1 and 0 explicitly brings out the fact that the dynamical moves on the two sublattices are alike for the choice of these special relations between the rates. This is crucial for further analysis.

In this new representation, a configuration $C$ is specified by the occupations of all sites. The time evolution of the probability $P(C)$ of the occurrence of $C$ is given by the master equation

$$
\frac{d P(C)}{d t}=\sum_{C^{\prime}} W\left(C^{\prime} \rightarrow C\right) P\left(C^{\prime}\right)-W\left(C \rightarrow C^{\prime}\right) P(C),
$$


where the transition rates $W\left(C \rightarrow C^{\prime}\right)$ are given by $p_{2}$ and $p_{1}$ for changes of configurations involving moves $(a)$ or $(b)$, and $(\bar{a})$ or $(\bar{b})$, respectively.

The dynamics preserves the sublattice densities $\rho_{1}^{o}$ and $\rho_{2}^{o}$. Within the subspace labeled by $\left(\rho_{1}^{o}, \rho_{2}^{o}\right)$, one can see that the system is ergodic by noting that the dynamics induces a leftward drift of a pairs 00 and 11 with rate $p_{2}$, and a rightward drift of such pairs with rate $p_{1}$. By successive application of moves (a) and (b) in Eqs. (9) and (10), and their reverses, any configuration $C$ in the subspace can be taken to a "standard configuration" $C_{o}$ in which all 1 's and all 0 's are clustered together. Since the lattice is periodic, the 11 and 00 pairs can be shifted to any other configuration $C^{\prime}$ from $C_{o}$. Thus any configuration $C$ can be taken to any configuration $C^{\prime}$ via $C_{o}$.

In steady state the right-hand side of Eq. (13) must vanish. A sufficient condition for this is that fluxes balance in pairs, i.e., for every flux out of a configuration $C$ to a configuration $C^{\prime}$, there should be an incoming flux from another uniquely determined configuration $C^{\prime \prime}$ into $C$. This is the condition of pairwise balance [8],

$$
W\left(C^{\prime \prime} \rightarrow C\right) P\left(C^{\prime \prime}\right)=W\left(C \rightarrow C^{\prime}\right) P(C),
$$

which is a generalization of the well-known condition of detailed balance [9].

For our problem, $C^{\prime \prime}$ may be constructed as follows. Let us denote the configuration $C$ symbolically as $1^{m_{1}} 0^{m_{2}} 1^{m_{3}} 0^{m_{4}} 1^{m_{5}} \cdots 0^{m_{k}}$, where there is a cluster of 1 's of size $m_{1}$, followed by a cluster of 0 's of length $m_{2}$, and so on, with a total of $k$ such clusters. Consider a transition to a configuration $C^{\prime} \equiv 1^{m_{1}} 0^{m_{2}-1} 1^{2} 01^{m_{3}-2} 0^{m_{4}} 1^{m_{5}} \ldots 0^{m_{k}}$ [a pair of 11 's jump to the left, i.e., move $(b)]$. One can always find a unique configuration, e.g., $C^{\prime \prime}$ $\equiv 1^{m_{1}} 0^{m_{2}} 1^{m_{3}-2} 01^{2} 0^{m_{4}-1} 1^{m_{5}} \cdots 0^{m_{k}}$ that gives rise to $C$ [via move $(b)]$. If the outgoing transition involves a rearrangement at the left edge of a cluster, the incoming transition involves a rearrangement at the right edge of the same cluster. Such an identification is possible also for transitions involving moves $(a),(\bar{a})$, and $(\bar{b})$, and ensures that $W\left(C^{\prime \prime}\right.$ $\rightarrow C)=W\left(C \rightarrow C^{\prime}\right)$. Thus Eq. (14) is satisfied provided the steady state probabilities obey

$$
P(C)=P\left(C^{\prime \prime}\right)=\text { const. }
$$

This means that in steady state, every allowed configuration is equally likely. The constant appearing in Eq. (15) can be found on using the normalization condition $\Sigma P(C)=1$. If $N_{1} \equiv \rho_{1}^{o} L$ and $N_{2} \equiv \rho_{2}^{o} L$ are the number of particles on the two sublattices, the total number of configurations in sector $\left(\rho_{1}^{o}, \rho_{2}^{o}\right)$ is $\mathcal{N}=\left({ }^{L} C_{N_{1}}\right)\left({ }^{L} C_{N_{2}}\right)$, where ${ }^{N} C_{M}$ is the number of ways of choosing $M$ out of a total of $N$ objects, and hence $P(C)=1 / \mathcal{N}$.

In the thermodynamic limit $L, N_{1}$, and $N_{2} \rightarrow \infty$, with $\rho_{1}^{o}, \quad \rho_{2}^{o}$ held constant, $P(C)$ approaches the product measure form

$$
\prod_{i} p\left(\sigma_{i}\right) p\left(\tau_{i+1 / 2}\right)=\rho_{1}^{o N_{1}}\left(1-\rho_{1}^{o}\right)^{L-N_{1}} \rho_{2}^{o N_{2}}\left(1-\rho_{2}^{o}\right)^{L-N_{2}} .
$$

This form of the steady state holds also for a higherdimensional generalization of the model involving rules (a) and (b) and their reverses along the sites and bonds in the $d$ directions of a simple cubic lattice.

The product measure weight in the steady state implies that correlation functions on different sites decouple. This then allows the current of $\sigma$ particles,

$$
\begin{aligned}
J_{1}= & \left(p_{2}-p_{1}\right)\left\langle\frac{\left(1+\sigma_{i}\right)}{2} \frac{\left(1-\sigma_{i+1}\right)}{2} \frac{\left(1-\tau_{i+1 / 2}\right)}{2}\right\rangle \\
& +\left(p_{2}-p_{1}\right)\left\langle\frac{\left(1+\sigma_{i+1}\right)}{2} \frac{\left(1-\sigma_{i}\right)}{2} \frac{\left(1+\tau_{i+1 / 2}\right)}{2}\right\rangle,
\end{aligned}
$$

to be found explicitly,

$$
J_{1}=\left(p_{2}-p_{1}\right) \rho_{1}\left(1-\rho_{1}\right)\left(1-2 \rho_{2}\right) .
$$

The first term in Eq. (17) comes from a particle hopping between sites $i$ and $i+1$ in the absence of a particle at site $i+1 / 2$ while the second is for hopping in the presence of a particle at the site in between. A similar expression holds for the $\tau$ current $J_{2}$,

$$
J_{2}=\left(p_{2}-p_{1}\right) \rho_{2}\left(1-\rho_{2}\right)\left(1-2 \rho_{1}\right) .
$$

The product measure form also allows us to find the roughness exponent of an associated height model, where the height fields associated with $\left\{\sigma_{i}\right\}$ and $\left\{\tau_{i}\right\}$ are, respectively, $h_{1 i}=\sum_{k=1}^{i}\left(\sigma_{k}-\left\langle\sigma_{k}\right\rangle\right)$ and $h_{2 i}=\sum_{k=1}^{i}\left(\tau_{k}-\left\langle\tau_{k}\right\rangle\right)$. Fluctuations of the height field are characterized by the root-meansquare height difference $G_{1}(r)=\sqrt{\left\langle\left(h_{1 i+r}-h_{1 i}\right)^{2}\right\rangle}$, with $G_{2}(r)$ defined similarly in terms of $\left\{h_{2 i}\right\}$. Using the fact that $\left\langle\sigma_{0} \sigma_{k}\right\rangle=\left\langle\sigma_{0}\right\rangle\left\langle\sigma_{k}\right\rangle$ for $k \neq 0$ and 1 for $k=0$, we find $G_{1}(r)$ $=G_{2}(r) \sim r^{1 / 2}$. Thus the roughness exponent $\chi$ defined by the growth of the root-mean-squared height fluctuations is

$$
\chi=1 / 2
$$

for both height fields. Evidently, this value will also characterizes fluctuations of linear combinations of the height fields $h_{1}$ and $h_{2}$, which arise when we deal with mode coordinate fields in the next section.

We close this section with numerical results for correlation functions away from the pairwise balance (PB) locus. We set $r_{2}=p_{1}=0$, and investigate what happens if we move away from the PB locus $r_{1}=p_{2}$. We studied the spatial correlation function $C(r)=\left\langle\sigma_{i} \sigma_{i+r}\right\rangle-\left\langle\sigma_{i}\right\rangle\left\langle\sigma_{i+r}\right\rangle$ by Monte Carlo simulation and show our results for different values of $r_{1}$ and $p_{2}$ in Fig. 2. As expected, for the PB case $r_{1}=p_{2}$ $=1$ (empty triangles in Fig. 2), the correlation length is zero. Away from $\mathrm{PB}$, with $r_{1}=1 / 3$ and $p_{2}=1$, the three curves for $C_{11}, C_{22}$, and $C_{12}$ representing, respectively, the intrasublattice correlation functions for sublattices 1 and 2 and the intersublattice correlation function all decay differently, but 


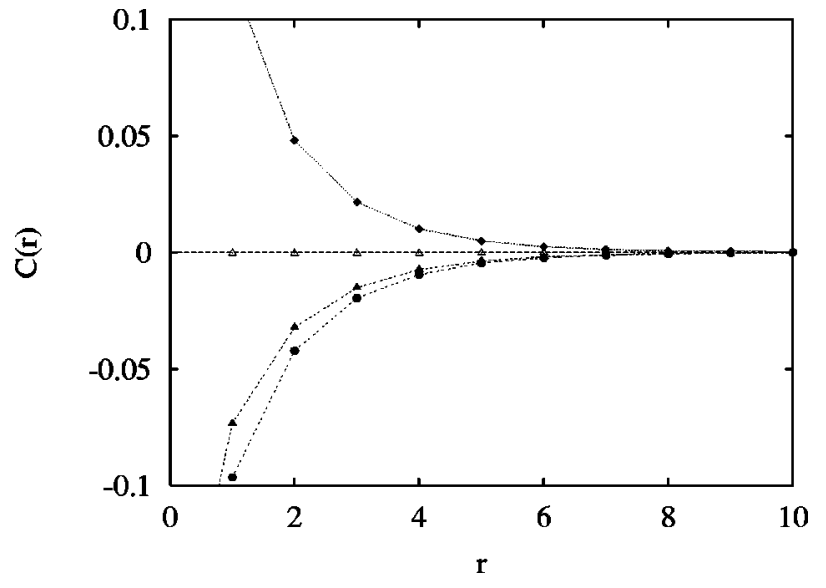

FIG. 2. The decay of correlation functions away from the line of pairwise balance is shown for $C_{11}$ (filled circle), $C_{22}$ (filled diamond), and $C_{12}$ (filled triangle). When pairwise balance does hold, the correlation function vanishes (empty triangles).

with a finite correlation length (Fig. 2). This shows that although product measure does not hold away from the pairwise balance locus $r_{1}=p_{2}$, correlations are short ranged so that the behavior on large length and time scales is expected to be similar to that in the pairwise balance case.

\section{B. Continuum equations and kinematic waves}

The expressions (18) and (19) for the current help us to construct approximate continuum equations for the evolution of the density fluctuation fields. The starting point is the pair of continuity equations

$$
\frac{\partial \rho_{m}}{\partial t}=-\frac{\partial}{\partial x} J_{m}\left(\rho_{1}, \rho_{2}\right), \quad m=1,2
$$

where $\rho_{1}(x, t)$ and $\rho_{2}(x, t)$ are coarse-grained densities at a mesoscopic scale and $J_{1}(x, t)$ and $J_{2}(x, t)$ are the corresponding currents. Each of these currents is written as the sum of three parts,

$$
J_{m}=J_{m}^{\text {systematic }}+J_{m}^{\text {diffusive }}+\eta_{m} .
$$

The systematic contributions $J_{m}^{\text {systematic }}$ at the mesoscopic scale are assumed to be given by the expressions (18) and (19) for the currents in an infinite system. The diffusive part $J_{m}^{\text {diffusive }}$ arises from local density inhomogeneities and is taken to be $-D_{m} \partial \rho_{m} / \partial x$. Finally $\eta_{m}$ is a noise term added to mimic stochasticity at the mesoscopic level; we consider uncorrelated white noise with $\left\langle\eta_{m}\right\rangle=0$ and $\left\langle\eta_{m}(x, t) \eta_{m}\left(x^{\prime}, t^{\prime}\right)\right\rangle=\Gamma \delta\left(x-x^{\prime}\right) \delta\left(t-t^{\prime}\right)$. These continuum equations have the same symmetries as the lattice model and hence would be expected to exhibit the same behavior on large length and time scales.

Writing $\rho_{1}=\rho_{1}^{o}+\tilde{\rho}_{1}$ and $\rho_{2}=\rho_{2}^{o}+\tilde{\rho}_{2}$ (where $\rho^{o}$,s are fixed average densities and $\tilde{\rho}$ 's are fluctuations) and using Eqs. (17), (19), (21), and (22), one can write down coupled equations governing the evolution of $\tilde{\rho}$ 's. We write these in terms of the height functions $h_{1}=\int{ }^{x} \tilde{\rho}_{1}\left(x^{\prime}, t\right) d x^{\prime}$ and $h_{2}$ $=\int^{x} \tilde{\rho}_{2}\left(x^{\prime}, t\right) d x^{\prime}$, the continuum analogs of the discrete functions $h_{1 i}$ and $h_{2 i}$ defined in the previous section. We find

$$
\begin{aligned}
\frac{\partial h_{1}}{\partial t}= & -r^{\prime}\left(1-2 \rho_{1}^{o}\right)\left(1-2 \rho_{2}^{o}\right) \frac{\partial h_{1}}{\partial x}+2 r^{\prime} \rho_{1}^{o}\left(1-\rho_{1}^{o}\right) \frac{\partial h_{2}}{\partial x} \\
& +D_{1} \frac{\partial^{2} h_{1}}{\partial x^{2}}+2 r^{\prime}\left(1-2 \rho_{1}^{o}\right) \frac{\partial h_{1}}{\partial x} \frac{\partial h_{2}}{\partial x}+r^{\prime}\left(1-2 \rho_{2}^{o}\right) \\
& \times\left(\frac{\partial h_{1}}{\partial x}\right)^{2}-2 r^{\prime}\left(\frac{\partial h_{1}}{\partial x}\right)^{2}\left(\frac{\partial h_{2}}{\partial x}\right)+\eta_{1}(x, t)
\end{aligned}
$$

and

$$
\begin{aligned}
\frac{\partial h_{2}}{\partial t}= & -r^{\prime}\left(1-2 \rho_{1}^{o}\right)\left(1-2 \rho_{2}^{o}\right) \frac{\partial h_{2}}{\partial x}+2 r^{\prime} \rho_{2}^{o}\left(1-\rho_{2}^{o}\right) \frac{\partial h_{1}}{\partial x} \\
& +D_{2} \frac{\partial^{2} h_{2}}{\partial x^{2}}+2 r^{\prime}\left(1-2 \rho_{2}^{o}\right) \frac{\partial h_{1}}{\partial x} \frac{\partial h_{2}}{\partial x}+r^{\prime}\left(1-2 \rho_{1}^{o}\right) \\
& \times\left(\frac{\partial h_{2}}{\partial x}\right)^{2}-2 r^{\prime}\left(\frac{\partial h_{2}}{\partial x}\right)^{2}\left(\frac{\partial h_{1}}{\partial x}\right)+\eta_{2}(x, t)
\end{aligned}
$$

where $r^{\prime}=\left(r_{1}-r_{2}\right)$.

Let us define $a=r^{\prime}\left(1-2 \rho_{1}^{o}\right)\left(1-2 \rho_{2}^{o}\right), \quad b=r^{\prime} \rho_{1}^{o}(1$ $\left.-\rho_{1}^{o}\right), \quad c=r^{\prime} \rho_{2}^{o}\left(1-\rho_{2}^{o}\right), \quad \kappa_{1}=r^{\prime}\left(1-2 \rho_{1}^{o}\right), \quad$ and $\quad \kappa_{2}=r^{\prime}(1$ $\left.-2 \rho_{2}^{o}\right)$. It is apparent that by taking linear combinations one can construct eigenmode fields $h_{ \pm}=\sqrt{c} h_{1} \pm \sqrt{b} h_{2}$, which decouple at the linear level. These fields describe wavelike modes [1] travelling with speeds $c_{ \pm}=-a \pm 2 \sqrt{b c}$. The time evolutions of these fields $h_{ \pm}$are governed by

$$
\begin{aligned}
\frac{\partial h_{+}}{\partial t}= & c_{+} \frac{\partial h_{+}}{\partial x}+D \frac{\partial^{2} h_{+}}{\partial x^{2}}+\frac{3}{2}\left(\frac{\kappa_{2}}{\sqrt{c}}+\frac{\kappa_{1}}{\sqrt{b}}\right)\left(\frac{\partial h_{+}}{\partial x}\right)^{2} \\
& +\left(\frac{\kappa_{2}}{\sqrt{c}}-\frac{\kappa_{1}}{\sqrt{b}}\right) \frac{\partial h_{+}}{\partial x} \frac{\partial h_{-}}{\partial x}-\frac{1}{2}\left(\frac{\kappa_{2}}{\sqrt{c}}+\frac{\kappa_{1}}{\sqrt{b}}\right)\left(\frac{\partial h_{-}}{\partial x}\right)^{2} \\
& -\frac{1}{2 \sqrt{b c}}\left(\frac{\partial h_{+}}{\partial x}\right)\left[\left(\frac{\partial h_{+}}{\partial x}\right)^{2}-\left(\frac{\partial h_{-}}{\partial x}\right)^{2}\right]+\eta_{+}(x, t), \\
\frac{\partial h_{-}}{\partial t}= & c_{-} \frac{\partial h_{-}}{\partial x}+D \frac{\partial^{2} h_{-}}{\partial x^{2}}+\frac{3}{2}\left(\frac{\kappa_{2}}{\sqrt{c}}-\frac{\kappa_{1}}{\sqrt{b}}\right)\left(\frac{\partial h_{-}}{\partial x}\right)^{2} \\
& +\left(\frac{\kappa_{2}}{\sqrt{c}}+\frac{\kappa_{1}}{\sqrt{b}}\right) \frac{\partial h_{+}}{\partial x} \frac{\partial h_{-}}{\partial x}-\frac{1}{2}\left(\frac{\kappa_{2}}{\sqrt{c}}-\frac{\kappa_{1}}{\sqrt{b}}\right)\left(\frac{\partial h_{+}}{\partial x}\right)^{2} \\
& -\frac{1}{2 \sqrt{b c}}\left(\frac{\partial h_{-}}{\partial x}\right)\left[\left(\frac{\partial h_{+}}{\partial x}\right)^{2}-\left(\frac{\partial h_{-}}{\partial x}\right)^{2}\right]+\eta_{-}(x, t) .
\end{aligned}
$$

The new noise terms $\eta_{ \pm}=\sqrt{c} \eta_{1} \pm \sqrt{b} \eta_{2}$, are also $\delta$ correlated. We have assumed $D_{1}=D_{2}=D$, though this may not be preserved in the effective long wavelength equations. The fields $h_{+}$and $h_{-}$are coupled at the nonlinear level so that each wave influences the dissipation of fluctuations of the 
other. We consider the dissipation properties of the waves in the next section for different sublattice filling fractions $\rho_{1}^{o}$ and $\rho_{2}^{o}$ of the sublattices.

\section{DISSIPATION OF THE WAVES AND DYNAMICAL EXPONENTS}

If it happens that some of the coefficients of Eq. (25) vanish for certain choices of densities $\rho_{1}^{o}$ and $\rho_{2}^{o}$, the evolution equations have special symmetries and this can have important implications for the long-time dynamics. As discussed below, there are three different symmetries that arise in the coupled-field problem, each corresponding to a different set of dynamical exponents. The dynamical exponents associated with the wave modes may differ. We have considered three special pairs of densities $\left(\rho_{1}^{o}, \rho_{2}^{o}\right)$ corresponding to three different symmetries.

\section{A. Symmetries of the equations}

To facilitate subsequent discussions let us first consider the case of a single field $h$ and list four different symmetries [10] for its evolution. (a) RI symmetry, invariance under updown reflection $(R)$ symmetry $h \rightarrow-h$ and under inversion (I) of space $x \rightarrow-x$; (b) RI symmetry, invariance under $h$ $\rightarrow-h$ and not under $x \rightarrow-x$; (c) $\overline{\mathrm{R}} \mathrm{I}$ symmetry, invariance under $x \rightarrow-x$ and not under $h \rightarrow-h$; (d) $\overline{\mathrm{R}} \overline{\mathrm{I}}$ symmetry, invariance neither under $x \rightarrow-x$, nor under $h \rightarrow-h$.

Since $\partial h / \partial t$ is an odd function of $h$, an equation of motion that contains only terms odd in $h$ will be said to have $R$ inversion symmetry, any term that is even in $h$ will be said to break $R$ symmtery. Accordingly: A term like $\partial^{2} h / \partial x^{2}$ obeys RI symmetry. Terms like $\partial h / \partial x$ and $(\partial h / \partial x)^{3}$ obey R⿳亠丷厂 symmetry. The $\bar{R} I$ symmetry is respected by the term $(\partial h / \partial x)^{2}$, while a term like $\partial h / \partial x$ added to it breaks that and gives rise to $\overline{\mathrm{R}} \overline{\mathrm{I}}$ symmetry.

To illustrate the occurrence of different types of symmetries in our coupled-field problem, we consider three special pairs of densities $\left(\rho_{1}^{o}, \rho_{2}^{o}\right)$.

(i) For $\rho_{1}^{o}=\rho_{2}^{o}=1 / 2$, Eq. (25) reduces to a pair of coupled equations with linear and first and second derivative terms and cubic gradient nonlinearities,

$$
\begin{aligned}
\frac{\partial h_{+}}{\partial t}= & 2 \sqrt{b c} \frac{\partial h_{+}}{\partial x}+\nu \frac{\partial^{2} h_{+}}{\partial x^{2}}-\frac{1}{2 \sqrt{b c}}\left(\frac{\partial h_{+}}{\partial x}\right)\left[\left(\frac{\partial h_{+}}{\partial x}\right)^{2}\right. \\
& \left.-\left(\frac{\partial h_{-}}{\partial x}\right)^{2}\right]+\eta_{+}(x, t), \\
\frac{\partial h_{-}}{\partial t}= & -2 \sqrt{b c} \frac{\partial h_{-}}{\partial x}+\nu \frac{\partial^{2} h_{-}}{\partial x^{2}}-\frac{1}{2 \sqrt{b c}}\left(\frac{\partial h_{-}}{\partial x}\right)\left[\left(\frac{\partial h_{+}}{\partial x}\right)^{2}\right. \\
& \left.-\left(\frac{\partial h_{-}}{\partial x}\right)^{2}\right]+\eta_{-}(x, t) .
\end{aligned}
$$

These equations describe two kinematic waves moving with speed $c_{+}=r^{\prime} / 2$ and $c_{-}=-r^{\prime} / 2$. The nonlinear couplings imply that each wave influences the evolution of the other. In order to study the dissipation of say the + mode, it is essential to move to the frame that comoves with it. This is accomplished by a Galilean shift $x \rightarrow x+c_{+} t$ and $t \rightarrow t$. In this frame, the evolution equations become

$$
\begin{aligned}
\frac{\partial h_{+}}{\partial t}= & \nu \frac{\partial^{2} h_{+}}{\partial x^{2}}-\frac{1}{2 \sqrt{b c}}\left(\frac{\partial h_{+}}{\partial x}\right)\left[\left(\frac{\partial h_{+}}{\partial x}\right)^{2}-\left(\frac{\partial h_{-}}{\partial x}\right)^{2}\right] \\
& +\eta_{+}(x, t), \\
\frac{\partial h_{-}}{\partial t}= & -4 \sqrt{b c} \frac{\partial h_{-}}{\partial x}+\nu \frac{\partial^{2} h_{-}}{\partial x^{2}}-\frac{1}{2 \sqrt{b c}}\left(\frac{\partial h_{-}}{\partial x}\right)\left[\left(\frac{\partial h_{+}}{\partial x}\right)^{2}\right. \\
& \left.-\left(\frac{\partial h_{-}}{\partial x}\right)^{2}\right]+\eta_{-}(x, t) .
\end{aligned}
$$

Evidently, in this frame, the - mode has a speed $c_{-}-c_{+}$ $=-4 \sqrt{b c}$. The pair of Eqs. (27) are invariant under $h_{+} \rightarrow$ $-h_{+}, \quad h_{-} \rightarrow-h_{-}$but not $x \rightarrow-x$, because of the linear $\partial h_{-} / \partial x$ and cubic nonlinear terms. The R⿳亠丷厂 symmetry holds in the rest frame of $h_{+}$mode.

Similarly the dissipation of the - mode can be studied by going to a frame that comoves with the - mode. It is easily seen that R⿳亠丷厂 symmetry holds in this frame as well.

Let us recall what happens when R $\bar{I}$ symmetry holds in the case of a single field $h$. In the Edwards-Wilkinson (EW) equation [11], the presence of an additional cubic term like $(\partial h / \partial x)^{3}$ reduces the RI symmetry to R⿳亠丷厂 symmetry. About the linear fixed point (with $\chi=1 / 2$ ), the cubic term has the same naive scaling dimension as $\left(\partial^{2} h / \partial x^{2}\right)$-both scale as $b^{-3 / 2}$ when $x \rightarrow b x$ and $h \rightarrow b^{\chi} h$. Such a marginal cubic term is known [12-15] to introduce logarithmic factors in the behavior of the height-height correlation functions. Using mode-coupling [12] and dynamical renormalization-group [13] calculations, it was found that the correlation function $F(t)=\sqrt{\left\langle[h(x, t)-h(x, 0)]^{2}\right\rangle}$ grows as $t^{1 / 4}[\ln (t)]^{1 / 8}$ and this was checked by Monte Carlo simulation. Recalling that $F(t) \sim t^{\beta}$ with $\beta=\chi / z$, we see that despite the lack of $I$ symmetry, the critical exponents $\beta$ and $z$ do not change from their EW values $1 / 4$ and 2, respectively.

In our case with two coupled fields, the cubic gradient terms again have the same naive scaling dimension as the linear second order term, but they are more complicated than just $(\partial h / \partial x)^{3}$. We might guess nevertheless due to the symmetry that each of the + and - modes have $z=2$. We will present numerical evidence in Sec. V B, which confirms this and shows that there are similar multiplicative logarithmic factors.

(ii) For $\rho_{1}^{o}=1 / 2$ and $\rho_{2}^{o} \neq 1 / 2$, the equations for the mode fields reduce to

$$
\begin{aligned}
\frac{\partial h_{+}}{\partial t}= & 2 \sqrt{b c} \frac{\partial h_{+}}{\partial x}+\nu \frac{\partial^{2} h_{+}}{\partial x^{2}}+\frac{3}{2} \frac{\kappa_{2}}{\sqrt{c}}\left(\frac{\partial h_{+}}{\partial x}\right)^{2}+\frac{\kappa_{2}}{\sqrt{c}} \frac{\partial h_{+}}{\partial x} \frac{\partial h_{-}}{\partial x} \\
& -\frac{1}{2} \frac{\kappa_{2}}{\sqrt{c}}\left(\frac{\partial h_{-}}{\partial x}\right)^{2}-\frac{1}{2 \sqrt{b c}}\left(\frac{\partial h_{+}}{\partial x}\right)\left[\left(\frac{\partial h_{+}}{\partial x}\right)^{2}-\left(\frac{\partial h_{-}}{\partial x}\right)^{2}\right] \\
& +\eta_{+}(x, t),
\end{aligned}
$$




$$
\begin{aligned}
\frac{\partial h_{-}}{\partial t}= & -2 \sqrt{b c} \frac{\partial h_{-}}{\partial x}+\nu \frac{\partial^{2} h_{-}}{\partial x^{2}}+\frac{3}{2} \frac{\kappa_{2}}{\sqrt{c}}\left(\frac{\partial h_{-}}{\partial x}\right)^{2} \\
& +\frac{\kappa_{2}}{\sqrt{c}} \frac{\partial h_{+}}{\partial x} \frac{\partial h_{-}}{\partial x}-\frac{1}{2} \frac{\kappa_{2}}{\sqrt{c}}\left(\frac{\partial h_{+}}{\partial x}\right)^{2}-\frac{1}{2 \sqrt{b c}}\left(\frac{\partial h_{-}}{\partial x}\right) \\
& \times\left[\left(\frac{\partial h_{+}}{\partial x}\right)^{2}-\left(\frac{\partial h_{-}}{\partial x}\right)^{2}\right]+\eta_{-}(x, t)
\end{aligned}
$$

Going to either of the frames in which the + mode or the - mode are at rest, we see that with the cubic nonlinearities, the $\overline{\mathrm{R}} \overline{\mathrm{I}}$ symmetry applies for each of the fields; $R$ symmetry is broken by by quadratic nonlinear terms and $I$ is broken because of linear first order and cubic terms. The most relevant terms at the linear fixed point are the quadratic nonlinear terms. Thus we would expect that these terms would govern the dissipation and give rise to the Kardar-ParisiZhang (KPZ) value $z=3 / 2$ [16] for both the modes.

(iii) For $\rho_{1}^{o}=\rho_{2}^{o} \neq 1 / 2$, we have $\kappa_{1}=\kappa_{2}$ and $c=b$, and the following pair of equations hold:

$$
\begin{aligned}
\frac{\partial h_{+}}{\partial t}= & c_{+} \frac{\partial h_{+}}{\partial x}+D \frac{\partial^{2} h_{+}}{\partial x^{2}}+3 \frac{\kappa_{2}}{\sqrt{c}}\left(\frac{\partial h_{+}}{\partial x}\right)^{2}-\frac{\kappa_{2}}{\sqrt{c}}\left(\frac{\partial h_{-}}{\partial x}\right)^{2} \\
& -\frac{1}{2 \sqrt{b c}}\left(\frac{\partial h_{+}}{\partial x}\right)\left[\left(\frac{\partial h_{+}}{\partial x}\right)^{2}-\left(\frac{\partial h_{-}}{\partial x}\right)^{2}\right]+\eta_{+}(x, t), \\
\frac{\partial h_{-}}{\partial t}= & c_{-} \frac{\partial h_{-}}{\partial x}+D \frac{\partial^{2} h_{-}}{\partial x^{2}}+2 \frac{\kappa_{2}}{\sqrt{c}} \frac{\partial h_{+}}{\partial x} \frac{\partial h_{-}}{\partial x}-\frac{1}{2 \sqrt{b c}}\left(\frac{\partial h_{-}}{\partial x}\right) \\
& \times\left[\left(\frac{\partial h_{+}}{\partial x}\right)^{2}-\left(\frac{\partial h_{-}}{\partial x}\right)^{2}\right]+\eta_{-}(x, t) .
\end{aligned}
$$

Here, an interesting situation arises. In the comoving frame of the - mode, the pair of equations are invariant under $h_{-} \rightarrow-h_{-}$and $h_{+} \rightarrow h_{+}$but not under $x \rightarrow-x$. Thus the $h_{-}$ field has $\mathrm{R} \overline{\mathrm{I}}$ symmetry, while the moving $h_{+}$field has $\overline{\mathrm{R}} \overline{\mathrm{I}}$ symmetry. The equations are invariant under $h_{-} \rightarrow-h_{-}$in any frame. The invariance under $x \rightarrow-x$ is broken (a) by the linear $\partial h_{ \pm} / \partial x$ and (b) by the trilinear terms. The effect of (a) can be shifted away by comoving; the (b) terms are expected to provide logarithmic multiplicative corrections. The same symmetries hold in the rest frame of the + wave. Based on these observations, we expect $z=2$ for the - mode (perhaps with multiplicative logarithmic corrections) and $z=3 / 2$ for the + mode. This is precisely the weak dynamical scaling discussed in the Introduction.

The quadratic nonlinear terms in Eq. (29) are exactly like those obtained in $[3,17]$. Similarly the nonlinearities in Eq. (28) are of the form obtained in [18]. The crucial difference is that we have additional linear gradient couplings, which bring in the possibility of observing weak dynamical scaling in our coupled-field system. We now turn to the next step of checking numerically and analytically our symmetry-based expectations.

\section{B. Growth exponents from Monte Carlo simulation}

For numerical simulation, we used a definition of height $\widetilde{h}_{i}(t)$ that differs slightly from $h_{i}(t)$ discussed so far. Instead of defining heights as density integrated over space with respect to a fixed site, we define $\widetilde{h}_{1 i}$ and $\widetilde{h}_{2 i}$ as integrated densities but with respect to the first particle, which is itself moving. Such a definition was used earlier in [19] and was found to markedly reduce the fluctuation in the height-height correlation function. If the particles on a particular sublattice are labeled $1, \ldots, N_{P}$, then the height $\tilde{h}_{i}(t)=n_{i}(t)$ $-\left\langle n_{i}(t)\right\rangle$ where $n_{i}(t)$ is the tag number of the particle at site $i$ and the subtracted part has a linear time dependence. If the site $i$ is empty, $\widetilde{h}_{i}$ is determined from the tags of the closest particles on either side by a lever rule. If $i_{o}(t)$ (whose average value $=v t$, with $v=$ particle speed) is the location of the particle 1 one has $\tilde{h}_{i}(t)=\sum_{k=i_{o}(t)}^{i} \tilde{\rho}_{k}-\rho^{o} i_{o}(t)$ using the fact that $n_{i}(t)=\sum_{k=i_{o}(t)}^{i}\left(\tilde{\rho}_{k}+\rho^{o}\right)$. In our problem, the heightheight correlation $\left\langle\widetilde{h}_{i A}(t)-\widetilde{h}_{i A}(0)\right\rangle$ grows as $-v_{A} \rho_{1}^{o} t$, where $v_{A}=\left(1-\rho_{1}^{o}\right)\left(1-2 \rho_{2}^{o}\right)$. The corresponding continuum equation for $\widetilde{h}_{1}(x, t)$ has two additional terms compared to Eq. (23), i.e., a constant $-v_{A} \rho_{1}^{o}$ that can be removed by an appropriate shift and a noise term $\zeta=v_{A} t \tilde{\rho}_{1}(x, t)$. The scaling dimension of $\zeta$ is lower $\left(\zeta \rightarrow b^{-z} \zeta\right)$ than the noise $\eta_{1}\left(\eta_{1}\right.$ $\rightarrow b^{-z / 2-1 / 2} \eta_{1}$ ) and hence is less relevant.

We are interested in knowing about the dissipation property of the \pm wave modes, so we numerically compute the height-height correlation function of the variable $\widetilde{h}_{ \pm}$in the comoving frame of the relevant mode, i.e., by a Galilean

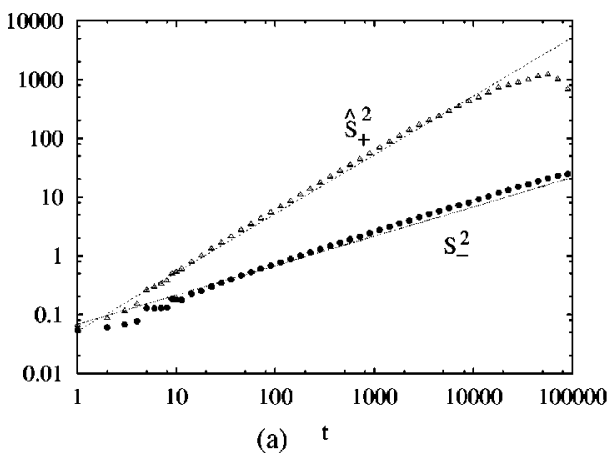

(a) $\mathrm{t}$

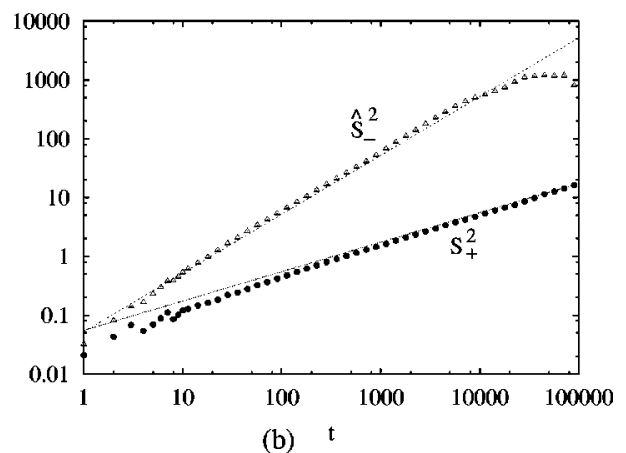

(b) $\mathrm{t}$
FIG. 3. Height correlation functions for $\rho_{1}^{o}=\rho_{2}^{o}=0.5 \quad(\mathrm{R} \overline{\mathrm{I}}$ symmetry) averaged over six histories. (a) In the rest frame of the - mode, $S_{-}^{2}$ grows as $\sim t^{1 / 2} \mathrm{im}$ plying $\beta=1 / 4$, while $\hat{S}_{+}^{2} \sim t$. (b) In the rest frame of the + mode, $S_{+}^{2}$ grows as $\sim t^{1 / 2}$, while $\hat{S}_{-}^{2} \sim t$. 


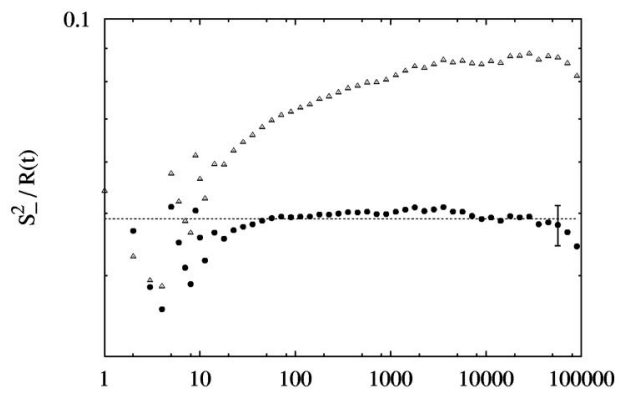

(a) $\mathrm{t}$

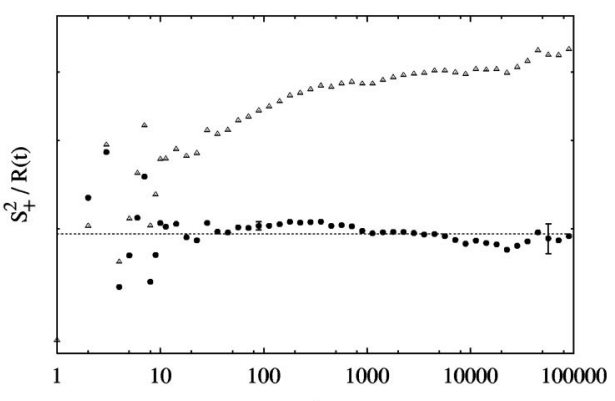

(b) ${ }^{t}$
FIG. 4. Effect of scaling the rest-frame height correlation functions of Fig. 3 by a factor $R(t)$ $=t^{1 / 2}$ (upper curves) and $R(t)$ $=t^{1 / 2}[\ln (t)]^{1 / 4} \quad$ (lower curves). The flattening of the latter confirms the presence of the multiplicative logarithms in both $S_{-}^{2}$ and $S_{+}^{2}$. shift with speed $c_{ \pm}$. The new fields $\widetilde{h}_{ \pm}$are the counterparts of $h_{ \pm}$defined earlier. We monitor the correlation functions

$$
S_{ \pm}^{2}(t)=\left\langle\left[\widetilde{h}_{ \pm}\left(x-c_{ \pm} t, t\right)-\tilde{h}_{ \pm}(x, 0)+J_{ \pm}^{o} t+\rho_{ \pm}^{o} c_{ \pm} t\right]^{2}\right\rangle
$$

where the average $\langle\cdots\rangle$ is both over space and the ensemble of configurations in the steady state, while $J_{ \pm}^{o}=\sqrt{c} v_{A} \rho_{1}^{o}$ $\pm \sqrt{b} v_{B} \rho_{2}^{o}$ and $\rho_{ \pm}^{o}=\sqrt{c} \rho_{1}^{o} \pm \sqrt{b} \rho_{2}^{o}$. We expect $S_{ \pm}^{2}(t)$ to grow as $t^{\beta}$ with $\beta=\chi / z$.

We used lattice sizes $L=54000$, while the number of history averages for each case is mentioned in the figure captions. There are two curves in each of the Figs. 3, 5, and 6 below, for example, in Fig. 3(b) the lower one represents $S_{+}^{2}$ and the upper one represents the height-height correlation function of the moving - mode in the rest frame of the + wave, namely, $\quad \hat{S}_{-}^{2}=\left\langle\left[\tilde{h}_{-}\left(x-c_{+} t, t\right)-\tilde{h}_{-}(x, 0)+J_{-}^{o} t\right.\right.$ $\left.\left.+\rho_{-}^{o} c_{+} t\right]^{2}\right\rangle$. Similarly $\hat{S}_{+}^{2}$ in Fig. 3(a) is defined in the rest frame of the - mode. The correlation functions $\hat{S}^{2}$,s increase linearly with time as they sense the effect of a moving kinematic wave. In a discrete lattice simulation, $x$ in Eq. (30) gets replaced by discrete integers $i$. The kinematic wave speeds are nevertheless real fractions, so height-height correlation functions $S^{2}$ and $\hat{S}^{2}$ have oscillations of the period $1 / c_{ \pm}$; these oscillations are noticeable for low $t$, but their relative contribution dies down for larger $t$.

(I) We first discuss the height-height correlations for $\rho_{1}^{o}$ $=0.5$ and $\rho_{2}^{o}=0.5$ (Fig. 3) corresponding to R⿳亠丷厂 symmetry. The kinematic waves go around the whole system with a time period $t_{o}=\left|L / c_{ \pm}\right|$. With $L=54000$ and $c_{ \pm}= \pm r^{\prime} / 2=$ $\pm 1 / 4$ we have $t_{o}=216000$ as $r^{\prime}=1 / 2$ in our simulations. The curve for the mode that is moving in the rest frame of the other should dip to a minimum at time $t_{o}$ and that is why it shows a flattening at times around $t=t_{o} / 2=108000$ when the moving wave has traveled halfway around.

Figure 3 gives strong evidence for $\beta=1 / 4$ for both modes in their respective rest frames when $\rho_{1}^{o}=\rho_{2}^{o}=1 / 2$. Thus $z$ $=\chi / \beta$ is equal to 2 for both modes. More careful examination of the data reveals that there are actually multiplicative logarithmic corrections to the leading power law behavior. The data is consistent with a growth $S(t) \sim t^{1 / 4}[\ln (t)]^{1 / 8}$, as may arise from cubic nonlinearities as discussed above. Evidence of this is seen in Figs. 4(a) and 4(b) which indicate that the data of Figs. 3(a) and 3(b) have factors $[\ln (t)]^{1 / 4}$ multiplying $t^{1 / 2}$. As discussed in Sec. V A, the latter are probably due to cubic nonlinearities.

(II) For $\rho_{1}^{o}=0.5$ and $\rho_{2}^{o}=1 / 3$, the system has R⿳亠口冋 symmetry. Figure 5 shows that both $S_{+}^{2}$ and $S_{-}^{2}$ grow as $\sim t^{2 / 3}$ implying that $\beta=1 / 3$ and $z=3 / 2$. Thus the dissipations of both the waves are KPZ-like.

(III) Finally Fig. 6 shows the height-height correlation functions for $\rho_{1}^{o}=1 / 3$ and $\rho_{2}^{o}=1 / 3$ (R $\overline{\mathrm{I}}$ symmetry for one mode, $\overline{\mathrm{R}} \overline{\mathrm{I}}$ symmetry for the other).

We see that $S_{-}^{2}$ grows as $\sim t^{1 / 2}$ with an indication of multiplicative logarithmic corrections, indicating $\beta=1 / 4$ while $S_{+}^{2}$ grows as $\sim t^{2 / 3}$ implying $\beta=1 / 3$. Recalling that $\chi=1 / 2$ for both modes, we have $z=2$ for the - mode and $z=3 / 2$ for the + mode. There is a logarithmic factor $[\ln (t)]^{1 / 4}$ multiplying $t^{1 / 2}$ for the - wave as is apparent from the flattening of the curve on dividing $S_{-}^{2}$ by $t^{1 / 2}[\ln (t)]^{1 / 4}$ (Fig. 7).

It is remarkable that although the waves are coupled nonlinearly to each other, two different dynamical exponents arise in the same system, in conformity with our expectations on symmetry grounds. This is the first instance we know of, in which such a property arises in a fully coupled system in which neither field evolves autonomously.

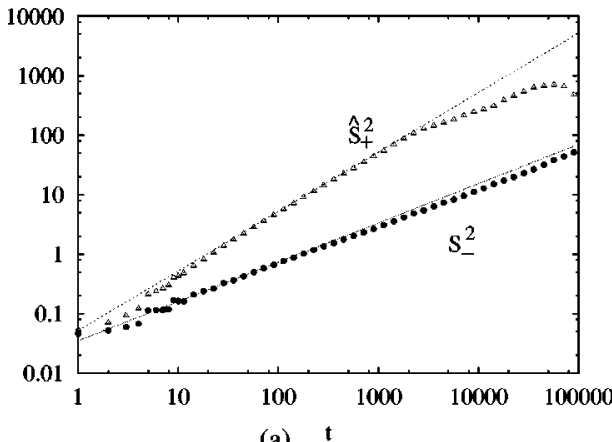

(a) $t$

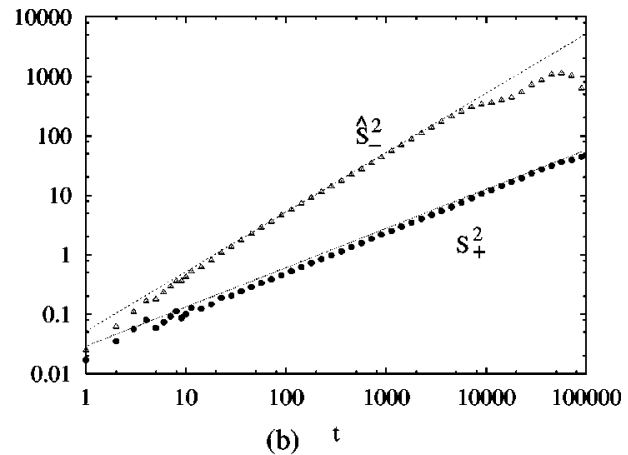

(b) $\mathrm{t}$
FIG. 5. Height correlation functions for $\rho_{1}^{o}=1 / 2, \rho_{2}^{o}$ $=1 / 3$ ( $\mathrm{R} \overline{\mathrm{I}}$ symmetry) averaged over four histories. (a) $S_{-}^{2}$ grows as $\sim t^{2 / 3}$ implying $\beta=1 / 3$, while $\hat{S}_{+}^{2} \sim t$. (b) $S_{+}^{2}$ too grows as $\sim t^{2 / 3}$ and $\hat{S}_{-}^{2} \sim t$. 


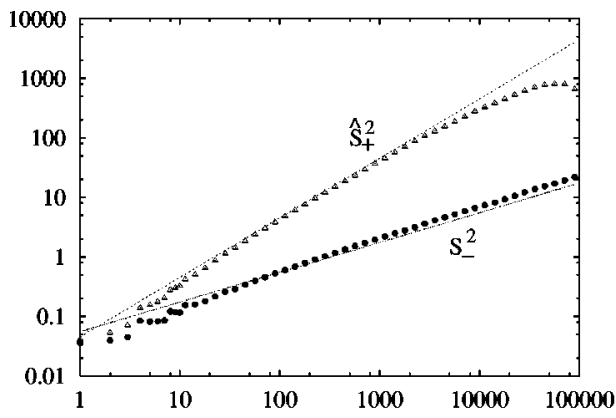

(a) $\mathrm{t}$

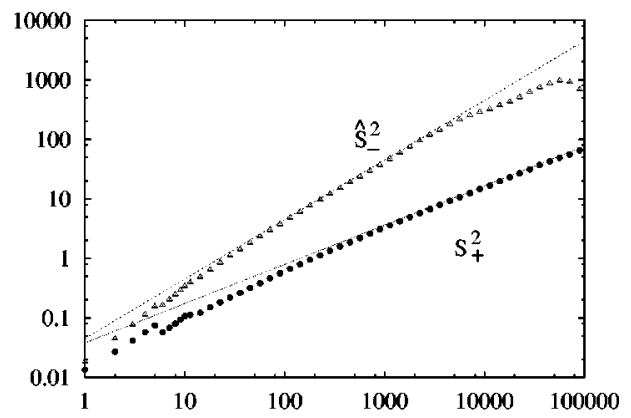

(b) $\mathrm{t}$
FIG. 6. Height correlation function for $\rho_{1}^{o}=\rho_{2}^{o}=1 / 3 \quad(\mathrm{R} \overline{\mathrm{I}}$ symmetry for one mode, $\overline{\mathrm{R}} \overline{\mathrm{I}}$ symmetry for the other) averaged over 12 histories. (a) $S_{-}^{2}$ grows as $\sim t^{1 / 2}$ and $\hat{S}_{+}^{2} \sim t$. (b) $S_{+}^{2}$ grows as $\sim t^{2 / 3}$ and $\hat{S}_{-}^{2} \sim t$.

\section{ANALYTICAL DEMONSTRATION OF WEAK DYNAMIC SCALING}

We argued in Sec. II in the context of the model equations (29) and showed by using Monte Carlo simulations that there are two different dynamical exponents $3 / 2$ and 2, respectively, for the two eigenmodes $h_{+}$and $h_{-}$in the model. In this section we use self-consistent mode-coupling and renormalization-group methods to study the large-distance, long-time properties of the correlation functions: We calculate the roughness exponents $\chi_{1,2}$ and the dynamical exponents $z_{1,2}$ of the fields $h_{+,-}$. Our analytical results agree with the previous numerical results. For appropriately chosen values for the densities of the particles in the lattice model [see above Eq. (29)], the continuum equations in the centerof-mass frame take the form

$$
\dot{h}_{+}-\frac{B_{o}}{2} \partial_{x} h_{+}+\frac{\lambda_{1}}{2}\left(\partial_{x} h_{+}\right)^{2}+\frac{\lambda_{2}}{2}\left(\partial_{x} h_{-}\right)^{2}=\nu_{+} \partial_{x x} h_{+}+f_{+},
$$

$$
\dot{h}_{-}+\frac{B_{o}}{2} \partial_{x} h_{-}+\lambda_{3}\left(\partial_{x} h_{+}\right)\left(\partial_{x} h_{-}\right)=\nu_{-} \partial_{x x} h_{-}+f_{-},
$$

where $\quad B_{o} / 2=c_{+}=-c_{-}, \quad \lambda_{1} / 2=3 \kappa_{2} / \sqrt{c}, \quad \lambda_{2} / 2=-\kappa_{2} / 2$,

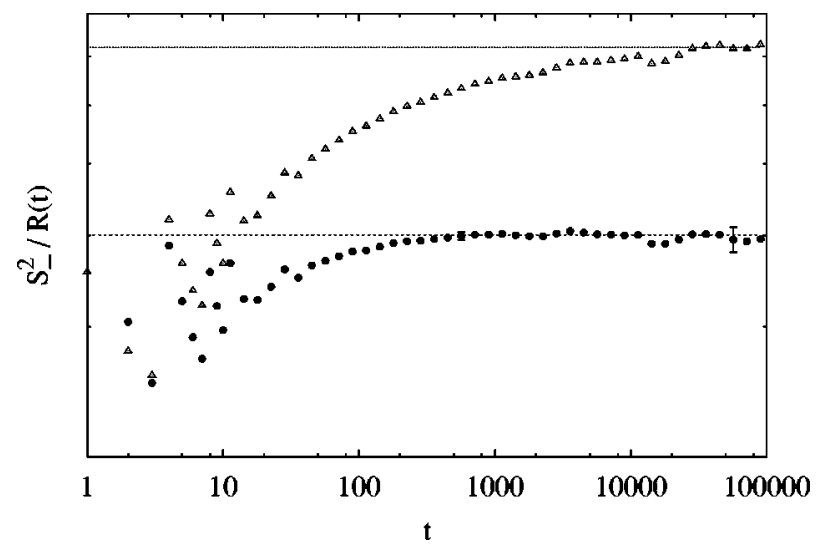

FIG. 7. Effect of rescaling $S_{-}^{2}$ in Fig. 6 by $R(t)=t^{1 / 2}$ (upper curve) and $R(t)=t^{1 / 2}[\ln (t)]^{1 / 4}$ (lower curve). The flattening of the latter confirms the presence of the logarithmic factor in $S_{-}^{2}$. $\lambda_{3}=2 \kappa_{2} / \sqrt{c}$. We denote the dissipation coefficients by $\nu_{1}$ and $\nu_{2}$ (we use two separate symbols in anticipation of weak dynamic scaling). We also ignore the cubic nonlinearities for simplicity. The waves can be removed from either of the Eqs. (31) separately by comoving with the left and right going waves through the Galilean shifts: $x \rightarrow x+\left(B_{o} / 2\right) t, \quad h_{+}$ $\rightarrow h_{+}$and $x \rightarrow x-\left(B_{o} / 2\right) t, \quad h_{-} \rightarrow h_{-}$, respectively. In the comoving frame of $h_{+}$, Eqs. (31) become

$$
\begin{gathered}
\dot{h}_{+}+\frac{\lambda_{1}}{2}\left(\partial_{x} h_{+}\right)^{2}+\frac{\lambda_{2}}{2}\left(\partial_{x} h_{-}\right)^{2}=\nu_{+} \partial_{x x} h_{+}+f_{+}, \\
\dot{h}_{-}+B_{o} \partial_{x} h_{-}+\lambda_{3}\left(\partial_{x} h_{+}\right)\left(\partial_{x} h_{-}\right)=\nu_{-} \partial_{x x} h_{-}+f_{-},
\end{gathered}
$$

whereas in the frame of $h_{-}$Eqs. (31) reduce to

$$
\begin{gathered}
\dot{h}_{+}-B_{o} \partial_{x} h_{h}+\frac{\lambda_{1}}{2}\left(\partial_{x} h_{+}\right)^{2}+\frac{\lambda_{2}}{2}\left(\partial_{x} h_{-}\right)^{2}=\nu_{+} \partial_{x x} h_{+}+f_{+}, \\
\dot{h}_{-}+\lambda_{3}\left(\partial_{x} h_{+}\right)\left(\partial_{x} h_{-}\right)=\nu_{-} \partial_{x x} h_{-}+f_{-}
\end{gathered}
$$

The bare response functions of the two fields $h_{+}$and $h_{-}$are given by

$$
G_{o}^{+}(k, \omega)=\frac{1}{i \omega+\nu_{+} k^{2}}, \quad G_{o}^{-}(k, \omega)=\frac{1}{i \omega-i B_{o} k+\nu_{-} k^{2}}
$$

in the right moving frame and

$$
G_{o}^{+}(k, \omega)=\frac{1}{i \omega+i B_{o} k+\nu_{+} k^{2}}, \quad G_{o}^{-}(k, \omega)=\frac{1}{i \omega+\nu_{-} k^{2}}
$$

in the left moving frame. The noise correlations in both the frames are given by

$$
\left\langle f_{+,-}(0,0) f_{+,-}(x, t)\right\rangle=2 D_{+,-} \delta(x) \delta(t) .
$$


Note that there is no frame in which the drift terms in both equations vanish. Noise correlations however do not change with change in reference frames as they are $\delta$ correlated in time.

In this section we analytically calculate the dynamic and roughness exponents $\chi_{i}, \quad z_{i}, i=+,-$ for the fields $h_{i}, \quad i=+,-, \quad$ respectively, defined by $\left\langle\left[h_{i}(x, t)\right.\right.$ $\left.\left.-h_{i}(0,0)\right]^{2}\right\rangle=x^{\chi_{i}} g\left(x^{z_{i}} / t\right)$. There are two limits in which their behavior is well understood analytically. First, in the absence of the kinematic wave $\left(B_{o}\right)$ terms, Eqs. (31) reduce to the Ertaş-Kardar [3] equations. Although the complete phase diagram of the latter is not known, they do have a locally stable renormalization-group fixed point belonging to the universality class of the KPZ equation [16], with $\chi_{+}$ $=\chi_{-}=1 / 2$ and $z_{+}=z_{-}=3 / 2$. Secondly, in the absence of the nonlinear terms coupling $h_{+}$and $h_{-}$, the kinematic wave terms can be removed separately in each equation by opposite Galilean transformations, yielding scaling properties independent of the wave speed. In the previous section our Monte Carlo results show that even when there is a nonlinear coupling, for particular densities, there is weak dynamic scaling: $z_{+}=3 / 2, \quad z_{-}=2$. Below, we offer an understanding of weak dynamic scaling in an analytical framework.

The possible occurence of weak dynamical scaling in this problem, makes a renormalization-group $(\mathrm{RG})$ treatment difficult: one cannot rescale time in two different ways for the two fields. We therefore adopt a self-consistent integralequation approach for which such weak scaling presents no difficulties. We then discuss how to circumvent the problems in applying an RG treatment.

\section{A. Self-consistent mode coupling calculation}

\section{KPZ equation in a moving frame}

Before embarking on a calculation for our model, it is instructive to look at the simpler case of a growing KPZ surface in a moving frame. The KPZ equation will be supplemented by a linear first order gradient term,

$$
\frac{\partial h}{\partial t}+c \frac{\partial h}{\partial x}+\lambda\left(\frac{\partial h}{\partial x}\right)^{2}=\nu \nabla^{2} h+\eta
$$

with $\langle\eta(x, t) \eta(0,0)\rangle=2 D \delta(x) \delta(t)$. We ask: Does the wave affect the exponents? We know the roughening exponent $\chi$ $=1 / 2$ for the KPZ equation in one dimension. Since $\chi$ reflects the static probability distribution of the heights, it is independent of reference frames. Due to the exponent identity $\chi+z=2[10]$, it follows that $z=3 / 2$. Thus in this particular case exponents are not affected by waves. The question is, can we see this in a self-consistent or renormalization-group calculation? Recall that the dynamic exponent is given by the width of the peak of the dynamic structure factor. The location of the peak, as a result of the wave induced by our transformation to a frame moving with speed $c$, is at $\omega=c k$. The response and the correlation functions in that frame are thus given by $G_{o}(k, \omega)=1 /(i \omega-i c k$ $\left.+\nu k^{2}\right)$ and $C_{o}(k, \omega)=2 D /\left[(\omega-c k)^{2}+\nu^{2} k^{4}\right]$, respectively. The one-loop integral that produces a singular correction to $\nu$ is

$$
I \sim-\left.\int d q d \Omega \frac{2 D q(k-q) k q}{\left[(\Omega-c k)^{2}+\nu^{2} q^{4}\right]\left[i(\omega-\Omega)-i c(k-q)+\nu(k-q)^{2}\right]}\right|_{\omega=c k} \sim-\int d q \frac{2 D q(k-q) k q}{\nu q^{2}\left[\nu q^{2}+\nu(k-q)^{2}\right]},
$$

which is the same as that one obtains in a standard calculation for the KPZ equation in the rest frame. A similar expression holds for the correction to the correlation function. In both these integrals, the wave can be shifted away trivially. Thus we obtain $z=3 / 2$ and $\chi=1 / 2$, in agreement with our expectations. With this background we now present the calculations for the exponents for our model. Interestingly, we will find that the effects of the waves cannot be trivially shifted away as they could in the simple example discussed above.

\section{B. Self-consistent calculation for the coupled model}

The mode-coupling approach to solving equations such as Eq. (31) consists in obtaining diagrammatic perturbation expansions for the renormalized propagator $G$ and correlation functions $C$ and resumming these in such a way that all the internal lines are renormalized correlation functions or propagators. This provides an exact solution for as asymptotically small wave number $q$ when the vertex corrections vanish for $q \rightarrow 0$ or due to some fundamental symmetry in the problem. In that case the problem is reduced to solving nonlinear integral equations for $G$ and $C$ whose order is the same as that of the nonlinearity in the modified Langevin equation.

In a one-loop self-consistent mode-coupling theory, one writes down one-loop integral equations for the response and correlation functions. The basic assumption is that there are one-loop corrections that diverge in the infrared limit. In a theory where there is no vertex renormalization due to some Ward-Takahashi identity (arising from some continuous symmetry of the equation of motion) these equations are exact, because any higher loop corrections can be incorporated within one-loop dressed response or correlation functions. However, when there is no such symmetry of the system to prevent vertex renormalization, the above assertion is not true. In such a situation, one also has to write down a oneloop self-consistent equation for the vertex, which now is to be solved simultaneously with the one-loop equations for response and correlation functions. An example of this has 

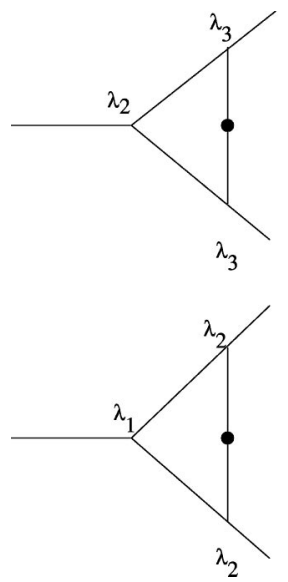
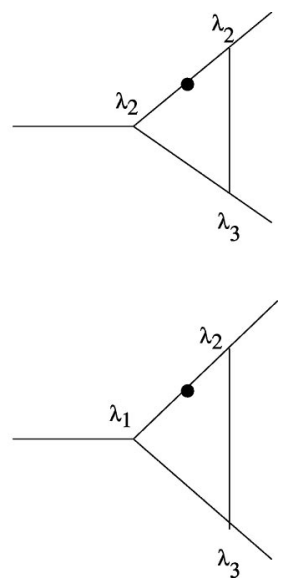

FIG. 8. One-loop diagrammatic corrections to $\lambda_{2}$; a line indicates a response function and a line with a small filled circle refers to a correlation function.

been worked out in [20]. Here we work with Eqs. (31). There are no diagramatic corrections to the $\lambda_{1}$ vertex at the oneloop level. However, there are no such conditions on $\lambda_{2}$ and $\lambda_{3}$. In our particular problem, however we show using bare response and correlation functions that one-loop vertex corrections for $\lambda_{2}$ and $\lambda_{3}$ are all infrared finite. Hence we ignore them in our calculations. In Figs. 8 and 9 we show the oneloop diagramatic corrections to $\lambda_{2}$ and $\lambda_{3}$, respectively. We first work in the comoving frame of $h_{+}$: In that frame $C_{+}(k, \omega)$ has a peak at $\omega=B_{o} k$ and $C_{-}(k, \omega)$ is peaked at $\omega=0$. Let us look at one of the diagrams very carefully (first diagram in Fig. 8),
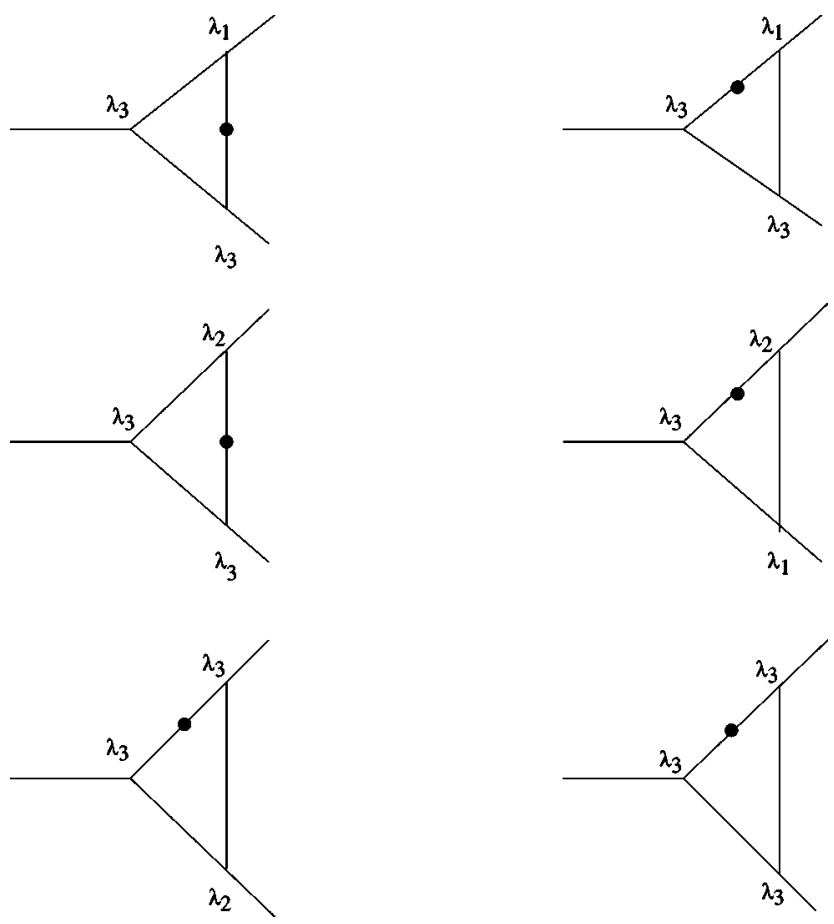

FIG. 9. One-loop diagrammatic corrections to $\lambda_{3}$; a line indicates a response function and a line with a small filled circle refers to a correlation function.
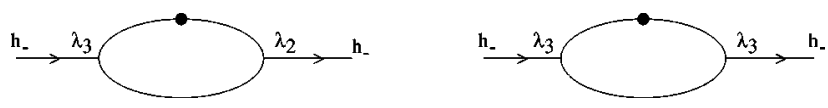

FIG. 10. One-loop diagrams contributing to $\Sigma_{-}(k, \omega)$. A line refers to a response function and a line with a small filled circle refers to a correlation function.

$$
\begin{aligned}
I & =i k \lambda_{2} \lambda_{3}^{2} / 2 \int \frac{d \Omega d q D_{+} q^{2} q^{2}}{\left[\left(\Omega-B_{o} q\right)^{2}+\nu_{-}^{2} q^{4}\right]\left[\Omega^{2}+\nu_{+} q^{4}\right]} \\
& =\frac{2 i k \lambda_{2} \lambda_{3}^{2}}{B_{o}^{2}}\left(\frac{1}{\nu_{+}}+\frac{1}{\nu_{-}}\right) \int d q D_{+} .
\end{aligned}
$$

This has no infrared divergence. Similarly, all other diagrams in Figs. 8 and 9 are also finite in the infrared limit. We ignore all these finite corrections for $\lambda_{2}$ and $\lambda_{3}$, i.e., we ignore vertex corrections in our self-consistent analysis. We again justify this a posteriori by showing that the vertex corrections remain finite in the self-consistent theory.

The time-displaced correlation functions of the fields $h_{+}$ and $h_{-}$are

$$
\begin{aligned}
& C_{+}(r, t) \equiv\left\langle h_{+}(0,0) h_{+}(r, t)\right\rangle, \\
& C_{-}(r, t) \equiv\left\langle h_{-}(0,0) h_{-}(r, t)\right\rangle .
\end{aligned}
$$

The scaling forms for the correlation and response functions as a function of wave number $k$ and frequency $\omega$ are

$$
\begin{aligned}
& C_{+}(k, \omega)=k^{-1-2 \chi_{+}-z_{+}} f_{+}\left(k^{z+} / \omega\right), \\
& C_{-}(k, \omega)=k^{-1-2 \chi_{-}-z_{-}} f_{-}\left(k^{z_{-}} / \omega\right),
\end{aligned}
$$

and

$$
\begin{aligned}
& G_{+}(k, \omega)=k^{-z_{+}} g_{+}\left(k^{z+} / \omega\right), \\
& G_{-}(k, \omega)=k^{-z_{-}} g_{-}\left(k^{z}-/ \omega\right) .
\end{aligned}
$$

Here, $z_{+}$are $z_{-}$are the dynamic exponents and $\chi_{+}$and $\chi_{-}$ are the roughness exponents of the fields $h_{+}$and $h_{-}$. Notice that we have allowed the existence of two different dynamic exponents. Since the fields are decoupled in the linearized theory, there is no cross propagator. The following one-loop diagrams contribute to the respective self-energies $\Sigma_{+}(k, \omega)$ and $\Sigma_{-}(k, \omega)$ (defined by $G_{i}^{-1}=G_{i 0}^{-1}-\Sigma_{i}$, where $G_{i 0}$ is the bare propagator for $\left.h_{i}, \quad i=+,-\right)$ of the fields $h_{+}$and $h_{-}$.

Notice that $G_{+0}^{-1}(k, \omega=0)=i B_{o} k+\nu_{+} k^{2}, \quad G_{-0}^{-1}=\nu_{-} k^{2}$. A self-consistent calculation is required if one encounters infrared divergences in the bare perturbation theory. The following one-loop diagrams in Figs. 10 and 11 contribute to $\Sigma_{-}(k, \omega)$ and $C_{-}(k, \omega)$, respectively. It is easy to see that none of these diagrams diverge in a bare perturbation theory. The first diagram in Fig. 10 has the form 


$$
\begin{aligned}
\Sigma_{-}(k, \omega) & \sim \lambda_{2} \lambda_{3} k \int d q \frac{D_{-} q^{2}(k-q)}{\nu_{+} q^{2}\left[\nu_{+} q^{2}+\nu_{-}(k-q)^{2}-i B_{o}(k-q)\right]} \\
& \sim \lambda_{2} \lambda_{3} k \int d q \frac{D_{-} q^{2}(k-q)\left[\nu_{+} q^{2}+\nu_{-}(k-q)^{2}\right]}{\nu_{-} q^{2}\left[\left\{\nu_{+} q^{2}+\nu_{-}(k-q)^{2}\right\}^{2}+B_{0}^{2}(k-q)^{2}\right]} \sim \lambda_{2} \lambda_{3} k \int d q \frac{D_{-}(k-q)}{\nu_{-} B_{0}^{2}(k-q)^{2}} \sim \text { finite, }
\end{aligned}
$$

since in the long wavelength limit $B_{0}^{2}(k-q)^{2}$ dominates over $\left\{\nu_{+} q^{2}+\nu_{-}(k-q)^{2}\right\}^{2}$. We have considered only the real part of $\Sigma_{-}(k)$ (only this part will renormalize $\nu_{-}$). However this does not produce any infrared singular correction to $\nu_{2}$ and hence we ignore it. Note that it is the presence of the kinematic waves that makes this diagrammatic contribution infrared finite and thus ignorable. The second diagram in Fig. 10 also has a similar finite form and is ignored again. In Fig. 11 the diagram has the form

$$
\begin{aligned}
& C_{-}(k, \omega) \\
& \quad \sim \frac{1}{k^{4}} \int \frac{d q q^{2}(k-q)^{2}}{q^{2}(k-q)^{2}\left[\nu_{-} q^{2}+\nu_{+}(k-q)^{2}-i B_{o}(k-q)\right]} .
\end{aligned}
$$

It is easy to see that this is not infrared divergent. So we ignore all corrections to $C_{-}(k, \omega)$. We immediately obtain $z_{-}=2, \quad \chi_{-}=1 / 2$. Note that the presence of the wave term $B_{0} k$ in the inverse propagator was again crucial to this analysis. We see from the foregoing analysis that

$$
G_{-}^{-1}(k, \omega) \sim i \omega+k^{2}, \quad C_{-}(k, \omega=0) \sim \frac{1}{\omega^{2}+k^{4}} .
$$

We now calculate the exponents of $h_{+}$. As stated earlier we ignore corrections to $\lambda_{2}$ and $\lambda_{3}$ as they are all finite. There are however, diverging one-loop corrections to both $C_{+}$and $\Sigma_{+}$that are shown in Figs. 12 and 13. Figures 12(a) and 13(a) come from the KPZ nonlinearity. If these were the only diagrams, one would have obtained KPZ exponents: $z_{+}=3 / 2, \quad \chi_{+}=1 / 2$. We notice, however, that Figs. 12(b) and 13(b) are as strongly infrared (IR) divergent as Figs. 12(a) and 13(a). Let us examine Fig. 12(b) in detail. The integral is given by (using the fact that $\chi_{-}=1 / 2, \quad z_{-}=2$ )

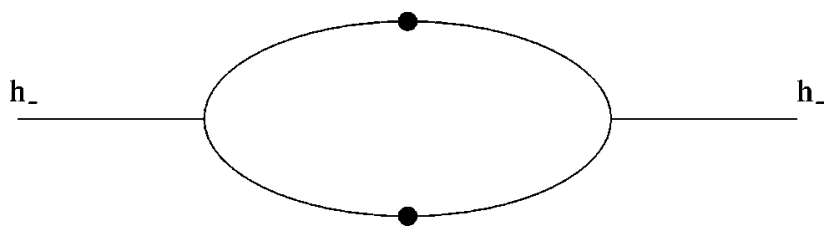

FIG. 11. One-loop contribution to $C_{2}(k, \omega)$. A line refers to a response function and a line with a small filled circle refers to a correlation function.

$$
\begin{aligned}
I \sim k \int d \Omega d q \frac{q^{2}(k-q)}{\left(\Omega^{2}+\nu_{-}^{2} q^{4}\right)\left[-i \Omega+i B_{o} k+\nu_{-}(k-q)^{2}\right]} \\
\sim k \int d q \frac{(k-q)\left[\nu_{-} q^{2}+\nu_{-}(k-q)^{2}\right]}{\nu_{-}\left[B_{o}^{2} k^{2}+\left\{\nu_{-} q^{2}+\nu_{-}(k-q)^{2}\right\}^{2}\right]} \\
\sim k \int d q \frac{(k / 2-q) \nu_{-} q^{2}}{\nu_{-}\left[B_{o}^{2} k^{2}+4 \nu_{-}^{2} q^{4}\right]} \sim \frac{k^{2}}{\nu_{-}} \int_{\sqrt{k}} \frac{d q}{q^{2}} \\
\quad \times\left[1+O\left(k^{2} / q^{4}\right)+\cdots\right] \sim k^{3 / 2} .
\end{aligned}
$$

Note that the presence of the kinematic wave was crucial again in this evaluation: The integral in Eq. (46) diverges as $\int d q / q^{2}$ if the external wave number $k$ is set to zero. At nonzero $k$, the integral is controlled by the presence of the wave term $B_{0}^{2} k^{2}$ in the denominator, and scales as $k^{-1 / 2}$, not as $k^{-1}$ as might have naively been expected. Thus $z_{+}=3 / 2$ is unaltered by the second diagram. Similarly we consider Fig. 13(b) (using $z_{+}=3 / 2$ ),

$$
\begin{aligned}
& I \sim \frac{1}{k^{3}} \int d \Omega d q \frac{q^{4}}{\left(\Omega^{2}+\nu_{-}^{2} q^{4}\right)\left[\left(\Omega-B_{o} k\right)^{2}+\nu_{-}^{2} q^{4}\right]} \\
& \sim \frac{1}{i \nu_{-} k^{3} B_{o} k} \int d q q^{2}\left[\frac{1}{B_{o} k-i \nu_{-} q^{2}}-\frac{1}{B_{o} k-i \nu_{-} q^{2}}\right] \sim k^{-7 / 2},
\end{aligned}
$$

which is as divergent Fig. 13(a). Thus $\chi_{+}=1 / 2$ also remains unaltered. It is easy to see that with these self-consistent response and correlation functions, one-loop corrections to $\lambda_{2}, \lambda_{3}$ do not diverge. Hence ignoring $\lambda_{2}, \lambda_{3}$ in our selfconsistent calculation is justified.

So far we have worked in the comoving frame of $h_{-}$, i.e, with Eqs. (33). Let us now go to the comoving frame of $h_{+}$: We work with Eqs. (32). The relevant diagrams are same as given in Figs. 12 and 13. It is easy to carry out a selfconsistent analysis again on Eqs. (32). The only difference is that now $C_{+}(k, \omega)$ is peaked at $\omega=0$ whereas $C_{-}(k, \omega)$ is peaked at $\omega=-B_{o} k$. Here again there is no singular correction to the reponse and the correlation functions of the field $h_{-}$. Thus $z_{-}=2$ and $\chi_{-}=1 / 2$. There are however diverging corrections to $\nu_{1}$ and $D_{1}$, which are identical to those ob-

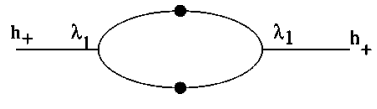

(a)

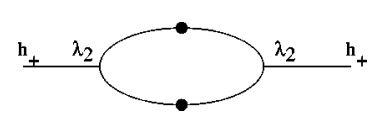

(b)
FIG. 12. One-loop contribution to $C_{+}(k, \omega)$. A line refers to a response function and a line with a small filled circle refers to a correlation function. 


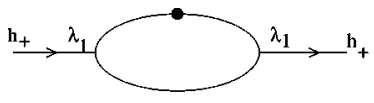

(a) (b)

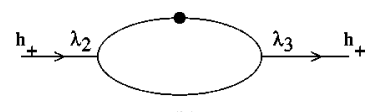

FIG. 13. One-loop contribution to $\Sigma_{+}(k, \omega)$. A line refers to a response function and a line with a small filled circle refers to a correlation function.

tained in our calculations in the comoving frame of $h_{-}$. Reassuringly, we find the same exponents again, i.e., $z_{+}$ $=3 / 2, \quad \chi_{+}=1 / 2$, as we should. Thus the effective equations in the left and right going frames are

$$
\begin{gathered}
\frac{\partial h_{+}(k)}{\partial t}+i B_{o} k h_{+}=\nu_{+} k^{3 / 2} h_{+}(k, t)+\cdots, \\
\frac{\partial h_{-}(k)}{\partial t}=\nu_{-} k^{2} h_{-}(k, t)+\cdots
\end{gathered}
$$

and

$$
\begin{gathered}
\frac{\partial h_{+}(k)}{\partial t}=\nu_{+} k^{3 / 2} h_{+}(k, t)+\cdots, \\
\frac{\partial h_{-}(k)}{\partial t}-i B_{o} k h_{-}=\nu_{-} k^{2} h_{-}(k, t)+\cdots .
\end{gathered}
$$

The renormalized equations (48) and (49) are thus connected by a Galilean transformation like the bare equations (33) and (32), and the exponents are frame independent.

\section{Renormalization-group analysis}

In Sec. V B we have shown how a self-consistent modecoupling treatment of our model for certain parameter values leads to weak dynamical scaling, i.e., distinct dynamical exponents for the two fields. In this section we recast these results within a perturbative dynamical renormalizationgroup (DRG) framework, and show how the difficulties posed for the DRG by weak dynamical scaling can be overcome.

The figures are same as shown in Sec. V B. We work with Eqs. (32). As usual, the bare diagrams for $\nu_{-}, D_{-}, \lambda_{2}$, and $\lambda_{3}$ do not diverge. Figure 12(a) diverges as $\sim\left[\left(D_{+} \lambda_{1}^{2}\right) / \nu_{1}^{3}\right] k^{2} \int d q / q^{2} \sim\left[\left(D_{+} \lambda_{1}^{2}\right) / \nu_{1}^{3}\right] k^{2}(1 / k), \quad$ whereas Fig. 12(b) diverges as $\lambda_{2} \lambda_{3} D_{-} k^{2} \int\left(d q / i B_{o} k+2 \nu_{-} q^{2}\right)$ $\sim \lambda_{2} \lambda_{3} D_{-} k^{2}(1 / \sqrt{k})$. Thus (b) can be ignored in comparison with (a) in the small $k$ limit. Similarly, Fig. 13(b) is less divergent than Fig. 13(a). Thus all relevant diagrams generating dominant singular corrections to the bare response and correlation functions of the field $h_{+}$are of KPZ type (with identical symmetry factors).

Starting with a cutoff wave number $\Lambda$, eliminating modes with wave numbers between $\Lambda e^{-\delta l}$ and $\Lambda$, rescaling so that the cutoff wave number is once more $\Lambda$, and passing to the limit $\delta l \rightarrow 0$, we obtain the differential recursion relations

$$
\begin{gathered}
\frac{d \nu_{+}}{d l}=\nu_{+}\left[z-2+\frac{g}{4}\right], \\
\frac{d D_{+}}{d l}=D_{+}\left[z-1-2 \chi_{+}+\frac{g}{4}\right],
\end{gathered}
$$

and

$$
\begin{gathered}
\frac{d \nu_{-}}{d l}=\nu_{-}[z-2], \\
\frac{d D_{-}}{d l}=D_{-}\left[z-1-2 \chi_{-}\right],
\end{gathered}
$$

where $\nu_{+}, \nu_{-}, D_{+}$, and $D_{-}$are now functions of $l$, and $g \equiv D_{+} \lambda_{1}^{2} / \nu_{+}^{3}$ is the dimensionless coupling constant. By using $g=2$ at the stable RG fixed point [10], we obtain

$$
\frac{d \nu_{+}}{d l}=\nu_{+}[z-3 / 2]
$$

$$
\frac{d \nu_{-}}{d l}=\nu_{-}[z-2]
$$

$$
\begin{aligned}
& \frac{d D_{+}}{d l}=D_{+}\left[z-1 / 2-2 \chi_{+}\right], \\
& \frac{d D_{-}}{d l}=D_{-}\left[z-1-2 \chi_{-}\right] .
\end{aligned}
$$

Now given the exponents $z, \chi_{+}, \chi_{-}$, the equations of motion (32) are supposed to be invariant under the scale transformations $\quad x \rightarrow b x, \quad t \rightarrow b^{z} t, \quad h_{+} \rightarrow b^{\chi_{+}} h_{+}, \quad h_{-} \rightarrow b^{\chi_{-}} h_{-}$. From Eqs. (50) we get $z=3 / 2, \chi_{+}=1 / 2$ whereas Eqs. (51) give $z=2, \chi_{-}=1 / 2$. Which value of $z$ should we choose? The choice of $z=3 / 2$ keeps $\nu_{1}$ fixed under rescaling, but $\nu_{2}(l)$ $\sim e^{-l / 2}$ as the RG fixed point is approached (remember $e^{l}$ is like a length scale). On the other hand if we choose $z=2$ then $\nu_{2}$ is fixed under rescaling but $\nu_{1} \sim e^{l / 2}$ as the fixed point is approached. Moreover, $z=2$ implies $\chi_{-}=1 / 2$ giving $C_{-}(x, 0) \sim x$ in agreement with the previously obtained results, and $\chi_{+}=1 / 4$ suggesting $C_{+}(x, 0) \sim x^{1 / 2}$, which, of course, is wrong. Similarly, the choice $z=3 / 2$ gives correct spatial dependence for $C_{+}(x, 0) \sim x$, but gives an incorrect result for $C_{-}(x, 0)$.

To resolve this difficulty, let us first study the seemingly trivial case of two totally decoupled fields,

$$
\begin{gathered}
\frac{\partial \phi_{1}}{\partial t}=\mu_{1} \frac{\partial^{2} \phi_{1}}{\partial x^{2}}+\eta_{1}, \\
\frac{\partial \phi_{2}}{\partial t}=-\mu_{2} \frac{\partial^{2} \phi_{2}}{\partial x^{4}}+\eta_{2},
\end{gathered}
$$

with $\left\langle\eta_{i}(0,0) \eta_{j}(x, t)\right\rangle=2 \gamma_{i} \delta_{i j} \delta(x) \delta(0)$ for $i=1,2$ and no sum on repeated indices. These are linear equations and hence exponents can be found exactly. In particular we know 


$$
\begin{aligned}
& \left\langle\phi_{1}(x, t) \phi_{1}(0,0)\right\rangle \sim x f_{1}\left(x^{2} / t\right), \\
& \left\langle\phi_{2}(x, t) \phi_{1}(0,0)\right\rangle \sim x^{3} f_{2}\left(x^{4} / t\right),
\end{aligned}
$$

i.e., we have $\chi_{1}=1 / 2, \quad \chi_{2}=3 / 2, \quad z_{1}=2, \quad z_{2}=4$. There are obviously no diagramatic corrections to any of the parameters. Here the flow equations are

$$
\begin{gathered}
\frac{d \mu_{1}}{d l}=\mu_{1}[z-2], \\
\frac{d \gamma_{1}}{d l}=\gamma_{1}\left[z-1-2 \chi_{1}\right], \\
\frac{d \mu_{2}}{d l}=\mu_{2}[z-4], \\
\frac{d \gamma_{2}}{d l}=\gamma_{2}\left[z-1-2 \chi_{2}\right] .
\end{gathered}
$$

A choice of $z=2$ and $\chi_{1}=1 / 2$ keeps $\mu_{1}$ and $\gamma_{1}$ fixed. This immediately tells us that the equal-time correlation function of the field $\phi_{1}$ scales as $A x^{2} \chi_{1}$. The coefficient $A$, a function of $\gamma_{1}$ and $\mu_{1}$, is scale independent. However a naive use of this value of $z$ (i.e., $z=2$ ) leads to a wrong conclusion about the spatial dependence of the equal-time correlation function of the field of $\phi_{2}: \quad z=2$ gives $\chi_{1}=1 / 2$ suggesting $\left\langle\phi_{2}(0,0) \phi_{2}(x, 0)\right\rangle \sim x$, which is wrong (the correct dependence is $x^{3}$, which is known exactly). The reason is that the choice $z=2$ makes the solutions of the flow equations for $\mu_{2}$ scale dependent: $\mu_{2} \sim e^{-2 l} \sim k^{2}$. However, one can still extract the correct behavior of the correlation function of $\phi_{2}$ in the above example if one accounts for the scale dependence $\mu_{2}$ explicitly: An explicit construction gives

$$
\begin{aligned}
& \left\langle\phi_{2}(k, \omega) \phi_{2}(-k, \omega)\right\rangle \\
& \equiv \frac{D_{2}}{\mu_{2} k^{1+2 \chi_{1}}}\left[\frac{1}{i \omega+\mu_{2} k^{z}}+\frac{1}{-i \omega+\mu_{2} k^{z}}\right],
\end{aligned}
$$

which gives (by using scale dependent $\mu_{2}$ ) $\left\langle\phi_{1}(0,0) \phi_{2}(x, 0)\right\rangle \sim x^{3}$, which is the correct answer. This suggests that to make sense of out the RG flow equations in presence of different dynamic scalings in a coupled system, one has to take care of the scale dependent diffusion coefficients while calculating the spatiotemporal behavior of the correlation functions correctly. Let us review our RG results obtained from the flow equations (50) and (51) in view of our previous analysis: We choose $z=2, \chi_{-}=1 / 2$, which gives $\chi_{+}=1 / 4$ and makes $\nu_{1} \sim k^{-1 / 2}$. Using this scale dependent coefficient we correctly obtain

$$
C_{+}(x, t)=x f_{-}\left[x^{3 / 2} / t\right]
$$

This suggests that an RG treatment, suitably modified, can be applied successfully to a problem of weak dynamic scaling provided scale dependent dissipation coefficients are taken into account while constructing the correlation functions.

\section{Strong dynamical scaling}

In Sec. II we have seen that for model equations (28) both the dynamic exponents turn out to be $3 / 2$ (i.e., KPZ-like). This is easy to understand analytically: In this case both the equations have KPZ-like nonlinearities in addition to the non-KPZ ones. However, it is easy to see that for both the fields, due to the presence of the waves, in any frame, nonKPZ diagrams are less singular than the corresponsing KPZlike diagrams. Thus the dominant singular corrections to the response and correlation functions for both the fields are KPZ-like, making $\chi_{+}=\chi_{-}=1 / 2$ and $z_{+}=z_{-}=3 / 2$.

\section{SUMMARY}

This paper is a study of the nature of spatiotemporal correlations in a coupled-field driven diffusive model (the LR model [4,5]), in the phase in which it displays kinematic waves. Our results include a demonstration of pairwise balance for certain parameter values and, hence, a proof that the steady state has a product measure in that range of parameters. Most important, we have been able to show that the fields corresponding to the two eigenmodes of the linearized version of the model were characterized by two different dynamic exponents, although the fields themselves are (nonlinearly) coupled. This is the first demonstration of such weak dynamical scaling in a model with two fully coupled fields (as distinct from the model of [3,18,20], where such an effective decoupling was found only in semiautonomously coupled systems in which one of the fields evolve independently). We have been able to show this numerically, through Monte Carlo simulations on a lattice model, and analytically, using self-consistent perturbative calculations as well as symmetry arguments, in the corresponding continuum stochastic PDEs. Outisde this special subspace of parameter space, the model exhibits normal, strong dynamic scaling. We also discuss and largely resolve the technical difficulties in applying the dynamical renormalization group when weak dynamic scaling prevails.

\section{ACKNOWLEDGMENT}

We thank Goutam Tripathy for valuable discussions.
[1] M. J. Lighthill and G. B. Whitham, Proc. R. Soc. London, Ser. A 229, 281 (1955).

[2] A. Basu, J. K. Bhattacharjee, and S. Ramaswamy, Eur. Phys. J. B 9, 425 (1999).
[3] D. Ertş and M. Kardar, Phys. Rev. E 48, 1228 (1993).

[4] R. Lahiri and S. Ramaswamy, Phys. Rev. Lett. 79, 1150 (1997).

[5] R. Lahiri, M. Barma, and S. Ramaswamy, Phys. Rev. E 61, 
1648 (2000).

[6] S. Ramaswamy, M. Barma, D. Das, and A. Basu, Report No. TIFR/TH/00-58; e-print cond-mat/0103062.

[7] D. Das and M. Barma, Phys. Rev. Lett. 85, 1602 (2000); D. Das, S. N. Majumdar, and M. Barma, e-print cond-mat/0102521.

[8] G. M. Schütz, R. Ramaswamy, and M. Barma, J. Phys. A 29, 837 (1996).

[9] N. G. van Kampen, Stochastic Processes in Physics and Chemistry (North-Holland, Amsterdam, 1992).

[10] A.-L. Barabási and H.E. Stanley, Fractal Concepts in Surface Growth (Cambridge University, Cambridge, 1995).

[11] S. F. Edwards and D. R. Wilkinson, Proc. R. Soc. London, Ser. A 381, 17 (1982).
[12] P. Devillard and H. Spohn, J. Stat. Phys. 66, 1089 (1992).

[13] M. Paczuski, M. Barma, S. N. Majumdar, and T. Hwa, Phys. Rev. Lett. 69, 2735 (1992)

[14] P. M. Binder, M. Paczuski, and M. Barma, Phys. Rev. E 49, 1174 (1994).

[15] B. Subramanian, G. T. Barkema, J. L. Lebowitz, and E. R. Speer, J. Phys. A 29, 7475 (1996).

[16] M. Kardar, G. Parisi, and Y. C. Zhang, Phys. Rev. Lett. 56, 889 (1986).

[17] M. Kardar, e-print cond-mat/9704172.

[18] A.-L. Barabási, Phys. Rev. A 46, R2977 (1992).

[19] G. Tripathy and M. Barma, Phys. Rev. E 58, 1911 (1998).

[20] A. K. Chattopadhyay, A. Basu, and J. K. Bhattacharjee, Phys. Rev. E 61, 2086 (2000). 